\title{
Analytical bond-order potential for the cadmium telluride binary system
}

\author{
D. K. Ward, ${ }^{1, *}$ X. W. Zhou, ${ }^{2}$ B. M. Wong, ${ }^{3}$ F. P. Doty, ${ }^{1}$ and J. A. Zimmerman ${ }^{2}$ \\ ${ }^{1}$ Radiation and Nuclear Detection Materials and Analysis Department, Sandia National Laboratories, Livermore, California 94550, USA \\ ${ }^{2}$ Mechanics of Materials Department, Sandia National Laboratories, Livermore, California 94550, USA \\ ${ }^{3}$ Materials Chemistry Department, Sandia National Laboratories, Livermore, California 94550, USA \\ (Received 27 September 2011; revised manuscript received 11 January 2012; published 22 March 2012)
}

\begin{abstract}
$\mathrm{CdTe}$ and $\mathrm{Cd}_{1-x} \mathrm{Zn}_{x}$ Te are the leading semiconductor compounds for both photovoltaic and radiation detection applications. The performance of these materials is sensitive to the presence of atomic-scale defects in the structures. To enable accurate studies of these defects using modern atomistic simulation technologies, we have developed a high-fidelity analytical bond-order potential for the CdTe system. This potential incorporates primary $(\sigma)$ and secondary $(\pi)$ bonding and the valence dependence of the heteroatom interactions. The functional forms of the potential are directly derived from quantum-mechanical tight-binding theory under the condition that the first two and first four levels of the expanded Green's function for the $\sigma$ - and $\pi$-bond orders, respectively, are retained. The potential parameters are optimized using iteration cycles that include first-fitting properties of a variety of elemental and compound configurations (with coordination varying from 1 to 12) including small clusters, bulk lattices, defects, and surfaces, and then checking crystalline growth through vapor deposition simulations. It is demonstrated that this CdTe bond-order potential gives structural and property trends close to those seen in experiments and quantum-mechanical calculations and provides a good description of melting temperature, defect characteristics, and surface reconstructions of the CdTe compound. Most importantly, this potential captures the crystalline growth of the ground-state structures for $\mathrm{Cd}$, $\mathrm{Te}$, and $\mathrm{CdTe}$ phases in vapor deposition simulations.
\end{abstract}

\section{INTRODUCTION}

CdTe and $\mathrm{Cd}_{1-x} \mathrm{Zn}_{x} \mathrm{Te}(\mathrm{CZT})$ crystals are very important semiconductor materials. As one example, CdTe is a leading semiconductor for terrestrial solar cell applications ${ }^{1,2}$ due to its manufacturability, high solar energy absorption coefficient, and optimal band gap for photoelectric conversion under solar radiation. ${ }^{3-5}$ Currently, CdTe modules have the lowest cost compared to any other photovoltaic technologies. ${ }^{6-8}$ As another example, CdTe-based CZT crystals have been the dominant semiconductor compounds for radiation detection applications. $^{9-12}$ This is a result of the material's high atomic numbers for efficient radiation-atomic interactions, and ideal band gaps for both a high electron-hole creation and a low leakage current.

While CdTe and CZT have been successfully applied in solar cell and radiation sensing fields, the possibility for further material improvement is still tremendous. In solar cell application, the record energy conversion efficiency currently achieved is only $\sim 16 \%$ as compared to the theoretical predictions of $29 \%$. $^{3,13-15}$ This difference is due to various micro/nanoscale defects in the multilayered films. ${ }^{5,8,13,16-18}$ In radiation detection applications, property nonuniformity results in both poor performance and high material cost (arising from a low yield of usable portions of ingots). ${ }^{9}$ The presence of micron-scale defects such as grain boundaries and tellurium inclusions/precipitates affects carrier transport and uniformity. ${ }^{9,19,20}$ These large defects are difficult to remove without understanding their formation mechanisms. Many smaller-scale defects also exist, such as vacancies, antisites, interstitials, and dislocations. ${ }^{21-23}$ It is unclear if minimizing only large defects will result in uniform transport properties because the presence of smaller defects, which have not been rigorously studied in the past, could also interact with charge carriers. In particular, experimental evidence has indicated that dislocations can directly affect charge carriers ${ }^{24,25}$ and can also serve as nucleation sites for tellurium precipitates. ${ }^{9}$ Clearly, an effective approach to study small-scale defects and ways of controlling them during growth can not only improve charge carrier properties directly but can also help limit nucleation of larger-scale defects.

Direct experimental observations of atomic-scale defects are extremely challenging. Recent advances in computational materials science technologies, including high-fidelity potentials ${ }^{26,27}$ and massively parallel molecular dynamic (MD) simulation codes (e.g., Large-Scale Atomic/Molecular Massively Parallel Simulator LAMMPS ${ }^{28,29}$ ), allow us to study configurations of atomic-scale defects and their formation mechanisms in detail. The key to high-fidelity MD simulations of CdTe or CZT is a highly transferrable (applicable for a variety of configurations) interatomic potential that accurately defines the interactions between various atoms. A literature survey indicates that two CdTe interatomic potentials are already developed: one ${ }^{30}$ is based upon the Stillinger-Weber (SW) formulation, ${ }^{31}$ and the other on ${ }^{32}$ is a Rockett modification ${ }^{33}$ of the Tersoff potential (TR). ${ }^{34}$ Our detailed analysis ${ }^{35}$ indicated that neither potential is sufficiently accurate at predicting properties necessary for understanding defects.

Bond-order potentials (BOPs) are based upon quantummechanical theories and can in principle offer a more accurate description of interatomic interactions compared to SW and Tersoff potentials. It is pointed out that Tersoff ${ }^{34}$ and Brenner $^{36}$ types of potentials can be viewed as a simple empirical BOP, building on the framework of empirical pseudopotentials, ${ }^{37}$ and considering only the $\sigma$ bonding with a second-moment approximation. ${ }^{38}$ To improve beyond 
Tersoff-Brenner potentials, a more advanced four-moment analytical BOP incorporating both $\sigma$ and $\pi$ bondings ${ }^{26,38-42}$ can be used. By analytically deriving the BOP from quantummechanical theory, its transferability to different phases can approach that of quantum-mechanical methods. The fidelity of BOP has been demonstrated in a number of previous studies. ${ }^{27,43} \mathrm{~A}$ valid potential format, however, does not itself ensure a high-quality potential without careful parameterization. ${ }^{44}$ One difficulty in parameterizing a complicated potential such as BOP is that because it is very flexible, any phase or configuration can have a large variation in energy with respect to a small change in parameters. Consequently, many phases must be specifically fitted to ensure that the ground-state (lowest-energy) phase matches that of experiments and quantum-mechanical calculations. While it is impossible to consider all possible configurations and difficult to ensure the inclusion of important phases during parameterization, simulations of melt- or vapor-phase growth can be used to test the validity of the parameterization as they sample a variety of local configurations statistically formed on a growth interface. More importantly, if any abnormal interfacial configuration has an incorrect energy lower than that of the equilibrium phase, it can trigger amorphous structures during the growth simulation of the equilibrium phase. Our previous study ${ }^{35}$ reveals that SW potentials can be easily parameterized to predict crystalline growth in vapor deposition simulations but at the cost of not predicting correct energy trends of different phases. In contrast, Tersoff potentials can more accurately capture the property trends, if correctly parameterized. However, Tersoff potentials are difficult to parameterize without checking crystalline growth using an iterative parameterization approach. Indeed, our simulations of vapor deposition confirmed that in the literature many Tersoff potentials ${ }^{32,45,46}$ predict amorphous growth. Yet, we also confirmed that both Tersoff potentials ${ }^{47,48}$ and BOP $^{27}$ can predict crystalline growth of semiconductor compounds in vapor deposition simulations ${ }^{43,49,50}$ if carefully parameterized.

The present work looks to develop a four-moment, $\sigma+\pi$ analytical BOP for the CdTe system that captures both property trends and crystalline growth; to describe a new procedure for improving Tersoff and BOP-like potential parameterization; to generate a comprehensive database characterizing our new CdTe BOP (including property trends of a variety of clusters and lattices for $\mathrm{Cd}$ and $\mathrm{Te}$ elements and CdTe compounds, as well as melting temperature, defect characteristics, and surface reconstructions of $\mathrm{CdTe}$ ); to validate the $\mathrm{CdTe} \mathrm{BOP}$ by comparing the database with the corresponding properties obtained from experiments and our high-level density functional theory (DFT) calculations; and to demonstrate the potential's ability of capturing crystalline growth of $\mathrm{Cd}$, Te elements, and CdTe compounds using vapor deposition simulations.

\section{BOND-ORDER POTENTIAL}

BOPs seek to improve over empirical potentials by formally coarse graining and linking the electronic structure inherent in a quantum-mechanical tight-binding model to atomic bonding. ${ }^{51}$ Pettifor and his collaborators ${ }^{26,39,40,52}$ have derived a general analytic expression for the BOP from tight-binding theory. In this theory, the bond energy is a multiplication of the bond-order and bond (hopping) integral, ${ }^{53}$ where the bond order is defined as one-half the difference between the number of bonding and antibonding electrons in the molecular orbitals between adjacent atoms, ${ }^{54}$ and the bond integral is related to the probability of an electron hopping from one molecular orbital to another and therefore depends on nearest-neighbor and orbital type. ${ }^{55}$ The BOP formalism includes separate contributions from $\sigma$ - and $\pi$-type bonds. ${ }^{39}$ The original BOP was derived for open-phase (half-filled valence shell) materials but later expanded to also include close-packed structures ${ }^{40}$ and compounds. ${ }^{38}$ The newest BOP has been generalized to incorporate the effects of valence shell filling, ${ }^{42}$ which enables the modeling of elements across the periodic table.

Retaining the previous nomenclature as much as possible, ${ }^{26,38-42}$ the total energy of a system containing $N$ atoms $(i=1,2, \ldots N)$ is expressed as

$$
\begin{aligned}
E= & \frac{1}{2} \sum_{i=1}^{N} \sum_{j=i_{1}}^{i_{N}} \phi_{i j}\left(r_{i j}\right)-\sum_{i=1}^{N} \sum_{j=i_{1}}^{i_{N}} \beta_{\sigma, i j}\left(r_{i j}\right) \cdot \Theta_{\sigma, i j} \\
& -\sum_{i=1}^{N} \sum_{j=i_{1}}^{i_{N}} \beta_{\pi, i j}\left(r_{i j}\right) \cdot \Theta_{\pi, i j},
\end{aligned}
$$

where $\phi_{i j}\left(r_{i j}\right)$ is a short-range two-body potential representing the overlap repulsion between a pair of ion cores, ${ }^{38} \beta_{\sigma, i j}\left(r_{i j}\right)$ and $\beta_{\pi, i j}\left(r_{i j}\right)$ are, respectively, $\sigma$ and $\pi$ bond integrals, $\Theta_{\sigma, i j}$ and $\Theta_{\pi, i j}$ are $\sigma$ and $\pi$ bond orders, which are functions of the local environment of atoms $i$ and $j$, and the list $j=i_{1}$, $i_{2}, \ldots, i_{N}$ represents neighbors of atom $i$. The repulsive energy $\phi_{i j}\left(r_{i j}\right)$ and the bond integrals $\beta_{\sigma, i j}\left(r_{i j}\right)$ and $\beta_{\pi, i j}\left(r_{i j}\right)$ are functions of the interatomic distance $r_{i j}$ between atom $i$ and $j . \phi_{i j}\left(r_{i j}\right), \beta_{\sigma, i j}\left(r_{i j}\right)$, and $\beta_{\pi, i j}\left(r_{i j}\right)$ are expressed in a general form as

$$
\begin{gathered}
\phi_{i j}\left(r_{i j}\right)=\phi_{0, i j} \cdot f_{i j}\left(r_{i j}\right)^{m_{i j}} \cdot f_{c, i j}\left(r_{i j}\right) \\
\beta_{\sigma, i j}\left(r_{i j}\right)=\beta_{\sigma, 0, i j} \cdot f_{i j}\left(r_{i j}\right)^{n_{i j}} \cdot f_{c, i j}\left(r_{i j}\right) \\
\beta_{\pi, i j}\left(r_{i j}\right)=\beta_{\pi, 0, i j} \cdot f_{i j}\left(r_{i j}\right)^{n_{i j}} \cdot f_{c, i j}\left(r_{i j}\right),
\end{gathered}
$$

where $\phi_{0, i j}, \beta_{\sigma, 0, i j}, \beta_{\pi, 0, i j}, m_{i j}$, and $n_{i j}$ are pair $(i j)$ dependent parameters, $f_{i j}\left(r_{i j}\right)$ is a Goodwin-Skinner-Pettifor (GSP) radial function, ${ }^{56}$ and $f_{c, i j}\left(r_{i j}\right)$ is a cutoff function. The GSP function is written as ${ }^{56}$

$$
f_{i j}\left(r_{i j}\right)=\frac{r_{0, i j}}{r_{i j}} \exp \left[\left(\frac{r_{0, i j}}{r_{c, i j}}\right)^{n_{c, i j}}-\left(\frac{r_{i j}}{r_{c, i j}}\right)^{n_{c, i j}}\right]
$$

with $r_{0, i j}, r_{c, i j}$, and $n_{c, i j}$ being pairwise parameters. The auxiliary cutoff function $f_{c, i j}\left(r_{i j}\right)$ is given in Appendix A.

The bond orders can be viewed as environment-dependent local variables that are $i j$ bond specific. ${ }^{26}$ The maximum value of the $\sigma$ bond order $\left(\Theta_{\sigma}\right)$ is 1 , while that of the $\pi$ bond order $\left(\Theta_{\pi}\right)$ is 2 , attributing to a maximum value of the total bond $\operatorname{order}\left(\Theta_{\sigma}+\Theta_{\pi}\right)$ of 3. The $\sigma$ and $\pi$ bond orders reflect the ubiquitous single-, double-, and triple-bond behavior of chemistry. Their analytical expressions can be derived from tight-binding theory by recursively expanding an intersite Green's function as a continued fraction. ${ }^{26,39}$ In particular, a Lanczos recursion algorithm $^{57,58}$ was used to derive the $\sigma$ bond order, and a matrix recursion ${ }^{59,60}$ was used to derive the $\pi$ bond order. To accurately represent the bonding with a computationally 
efficient potential formulation suitable for MD simulations, the derived BOP takes (and retains) the first two and the first four levels of the recursive representations for the $\sigma$ and $\pi$ bond orders, respectively, similar to Murdick et al. ${ }^{44}$

Bond-order terms can be understood in terms of molecular orbital hopping paths based upon the Cyrot-Lackmann theorem. ${ }^{26}$ In general, the $n$th moment of the electronic eigenspectrum can be represented by all hopping paths of length $n$ around the bond. ${ }^{61}$ These hopping paths naturally incorporate the effects of local configuration around the bond, including bond lengths and (three- and four-body) bond angles. Many possible hopping paths around a bond exist; the most important ones were determined and are shown in Fig. 1. These hopping paths are incorporated in the BOP as will be clear below.

Based on the approach described above, the two-level $\sigma$ bond order for the BOP approximation with a half-full valence shell is written as

$$
\Theta_{\sigma, i j}^{(1 / 2)}=\frac{\beta_{\sigma, i j}\left(r_{i j}\right)}{\sqrt{\beta_{\sigma, i j}^{2}\left(r_{i j}\right)+c_{\sigma, i j} \cdot\left[\beta_{\sigma, i j}^{2}\left(r_{i j}\right) \cdot \Phi_{2 \sigma}^{i}+\beta_{\sigma, i j}^{2}\left(r_{i j}\right) \cdot \Phi_{2 \sigma}^{j}\right]+\varsigma_{1}}},
$$

where $c_{\sigma, i j}$ is a pair-dependent empirical parameter that compensates for some of the error introduced by a two-level $\sigma$ bond-order approximation, $\Phi_{2 \sigma}^{i}$ and $\Phi_{2 \sigma}^{j}$ are local variables arising from the 2-hops shown in Fig. 1(a) whose expressions will be given below. Note that Eq. (6) is modified from previous expressions ${ }^{26,27}$ by multiplying the numerator and denominator by $\beta_{\sigma, i j}\left(r_{i j}\right)$ and retains unity in the absence of neighbors. To implement BOP in numerical MD codes, four small numbers $\varsigma_{n}$ ( $n=1-4$ represents an index number) are introduced. These numbers remove singularities (such as divide-by-zero or square root of negative values) but are small enough to virtually have no effect on the results. Detailed expressions for $\Phi_{2 \sigma}^{i}$ and $\Phi_{2 \sigma}^{j}$ are given in Appendix A.

Equation (6) pertains to a half-full valence shell. A general $\sigma$ bond-order term that includes valence shell occupancy can be extrapolated from the half-full bond order by using a combination of symmetric and asymmetric bond-order functions: ${ }^{27,42}$

$$
\Theta_{\sigma, i j}=\Theta_{s, i j}\left(\Theta_{\sigma, i j}^{(1 / 2)}, f_{\sigma, i j}\right) \cdot\left[1-\left(f_{\sigma, i j}-\frac{1}{2}\right) \cdot k_{\sigma, i j} \frac{\beta_{\sigma, i j}^{2}\left(r_{i j}\right) \cdot R_{3 \sigma, i j}}{\beta_{\sigma, i j}^{2}\left(r_{i j}\right)+\frac{\beta_{\sigma, i j}^{2}\left(r_{i j}\right) \cdot \Phi_{2 \sigma}^{i}+\beta_{\sigma, i j}^{2}\left(r_{i j}\right) \cdot \Phi_{2 \sigma}^{j}}{2}+\varsigma_{2}}\right],
$$

where $0 \leqslant f_{\sigma, i j} \leqslant 1$ is a pairwise band-filling parameter representing the number of electrons in the valence shell normalized by the full valence shell (eight electrons), $k_{\sigma, i j}$ is a pairwise skewing parameter, $R_{3 \sigma, i j}$ is a local variable arising from the three-member ring hops shown in Fig. 1(a), and $\Theta_{s, i j}$ is a symmetric band-filling function that modifies the half-full valence shell bond-order expression. The second term in Eq. (7) approximates the asymmetric eigenspectrum with a skewing term proportional to the normalized three-member ring contribution. Further expressions for $R_{3 \sigma, i j}$ and $\Theta_{s, i j}$ are included in Appendix A.

Based upon the derivation of Pettifor, ${ }^{26,38}$ the analytic expression for the $\pi$ bond order is expressed as

$$
\begin{aligned}
\Theta_{\pi, i j}= & \frac{a_{\pi, i j} \cdot \beta_{\pi, i j}\left(r_{i j}\right)}{\sqrt{\beta_{\pi, i j}^{2}\left(r_{i j}\right)+c_{\pi, i j} \cdot\left(\frac{\beta_{\pi, i j}^{2}\left(r_{i j}\right) \cdot \Phi_{2 \pi, i j}^{i}+\beta_{\pi, i j}^{2}\left(r_{i j}\right) \cdot \Phi_{2 \pi, i j}^{j}}{2}+\sqrt{\beta_{\pi, i j}^{4}\left(r_{i j}\right) \cdot \Phi_{4 \pi, i j}+\varsigma_{3}}\right)+\varsigma_{4}}} \\
& +\frac{a_{\pi, i j} \cdot \beta_{\pi, i j}\left(r_{i j}\right)}{\sqrt{\beta_{\pi, i j}^{2}\left(r_{i j}\right)+c_{\pi, i j} \cdot\left(\frac{\beta_{\pi, i j}^{2}\left(r_{i j}\right) \cdot \Phi_{2 \pi, i j}^{i}+\beta_{\pi, i j}^{2}\left(r_{i j}\right) \cdot \Phi_{2 \pi, i j}^{j}}{2}-\sqrt{\beta_{\pi, i j}^{4}\left(r_{i j}\right) \cdot \Phi_{4 \pi, i j}+\varsigma_{3}}+\sqrt{\varsigma_{3}}\right)+\varsigma_{4}}},
\end{aligned}
$$

where $c_{\pi, i j}$ and $a_{\pi, i j}$ are pairwise parameters. Equation (8) can be calculated from $\beta_{\pi, i j}^{2}\left(r_{i j}\right) \cdot \Phi_{2 \pi, i j}^{i}$ and $\beta_{\pi, i j}^{4}\left(r_{i j}\right) \cdot \Phi_{4 \pi, i j}$ corresponding, respectively, to the 2 - and 4-hops shown in Fig. 1(b). The expressions of these terms are given in Appendix A.

To summarize, the analytic BOP developed here is expressed in terms of bonding and repulsive energy components, Eq. (1). The bonding energy is split into $\sigma$ and $\pi$ bonding terms, each of which is a product of a bond integral and a bond order. The repulsive energy, Eq. (2), and the bond integrals, Eqs. (3) and (4), are approximated with two-body GSP functions. The $\sigma$ bond order with a half-full valence shell, Eq. (6), is used to extrapolate the bond-order expression that incorporates explicit valence band filling, Eq. (7). This $\sigma$ bond-order expression also contains a three-member ring term that allows implementation of an asymmetric density of states, which helps to either stabilize or destabilize close-packed structures. The $\pi$ bond order, Eq. (8), includes hopping paths of length 4. This enables the incorporation of effects of a dihedral angle as can be seen from Eq. (A10) in Appendix A.

\section{PARAMETERIZATION}

The BOP parameterization of CdTe can be done independently for elemental $\mathrm{Cd}$, elemental $\mathrm{Te}$, and finally for CdTe. As stated above, the ability to capture crystalline growth is a critical component of a high-fidelity interatomic potential. In general, a more transferrable (flexible for many 
(a) $\sigma$ bond

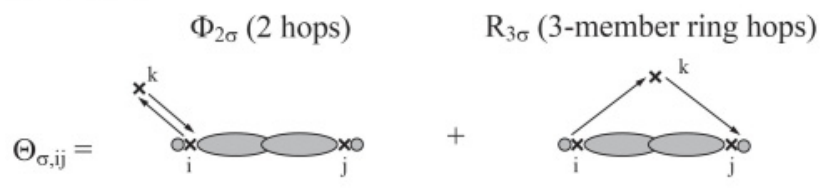

(b) $\pi$ bond

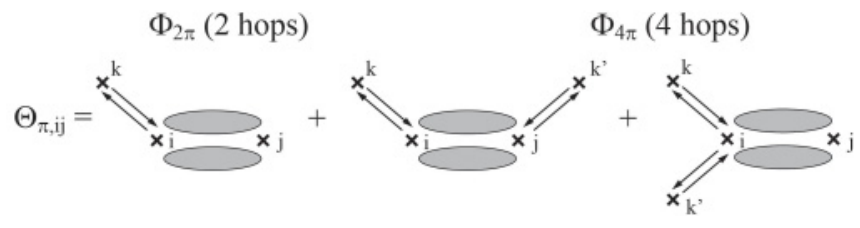

FIG. 1. A schematic representation of the hopping paths that sample the local configuration around the $i j$ bond: (a) the primary $\sigma$ bond and (b) the secondary $\pi$ bond.

phases) potential is more difficult to parameterize for capturing crystalline growth because the properties of various phases vary more dramatically with changes of the parameters. The parameterization of the sophisticated BOP is not trivial and hence is described in detail.

\section{A. Fitting parameters}

The CdTe binary BOP model includes a total of 69 parameters, as listed in Tables I through IV of Appendix B. However, many parameters can be fixed prior to the fitting process. First, the four global parameters $\varsigma_{1}-\varsigma_{4}$ are chosen to be small numbers as shown in Table I. Next, the characteristic bond lengths $r_{0}, r_{c}, r_{1}, r_{\text {cut }}$ are selected, as shown in Table III, based upon the criteria that $r_{0}$ approximately scales with the bond length of the corresponding atomic pair in the equilibrium structure, $r_{c}$ equals $r_{0}, r_{\text {cut }}$ roughly scales with $r_{0}$, and $r_{1}$ is midway between $r_{0}$ and $r_{\text {cut }}$. The pairwise parameters $c_{\pi}$ and $a_{\pi}$ are set to unity as were originally derived for the $\pi$ bond order. ${ }^{39,40}$ The CdTe zb crystal structure has a half-full valence shell and does not make use of the symmetric and asymmetric $\sigma$ bond-order terms; therefore, we set $f_{\sigma}=0.50$ and $k_{\sigma}=0$ for the Cd-Te bond. Finally, we set the three-body parameter $g_{0}$ to be the normalized value of unity for all the triples as shown in Table IV. This leaves 39 parameters to be determined.

\section{B. Target structures}

An effective approach to ensure a highly transferable, growth-simulation-enabling interatomic potential for semiconductors is to directly fit (or at least monitor) the atomic volumes, cohesive energies, and elastic properties of a correct set of target structures, including $\mathrm{Cd}, \mathrm{Te}$, and $\mathrm{CdTe}$ clusters and phases with coordination from 1 to 12 . After extensive trialand-error parameterization iterations with crystalline growth simulations included as part of the validation tests, we narrowed down our target structures to the following clusters and lattices: For clusters, we have dimer (di), trimer (tri), square (sq), rhombus (rhom), tetrahedron (tetra), and four-atom-chain (ch) for each of the three materials (element or compound) $\mathrm{Cd}$, $\mathrm{Te}$, and $\mathrm{CdTe}$, plus two nonstoichiometric trimers $\mathrm{Cd}_{2} \mathrm{Te}$ and $\mathrm{CdTe}_{2}$. For lattices, we have diamond-cubic (dc), simple-cubic (sc), body-centered-cubic (bcc), face-centered-cubic (fcc), hexagonal-close-packed (hcp), graphite (gra), and graphene (grap) phases for $\mathrm{Cd}$ and the same for Te with the addition of $\gamma$-Se (A8); zinc-blende (zb), wurtzite (wz), $\mathrm{NaCl}$ (B1), $\mathrm{CsCl}$ (B2), binary-graphite (bgra), binary-graphene (bgrap), $\mathrm{AuCu}$ (L1 $\left.1_{0}\right), \mathrm{CuPt}\left(\mathrm{L1}_{1}\right), \mathrm{NiAs}\left(\mathrm{B} 8_{1}\right), \mathrm{CrB}$ (B33), AlSb (sc16), and face-centered square (fcs) for the stoichiometric compound $\mathrm{CdTe}$; and $\mathrm{Ag}_{2} \mathrm{O}$ (cP4) and $\mathrm{ZrO}_{2}$ for the two nonstoichiometric compounds $\mathrm{CdTe}_{2}$ and $\mathrm{Cd}_{2} \mathrm{Te}$. Although all these phases are not used in a particular parameterization, monitoring the energies of many structures helps select the important ones and their weighting factors for fitting to ensure the lowest energies for the equilibrium phases. For example, the $\mathrm{L} 1_{0}, \mathrm{~L} 1_{1}$, and sc16 structures of the CdTe compound sometimes became more stable than the equilibrium zb phase with relatively small changes in the parameters.

\section{Two-step fitting method}

We discovered that a simple minimization of the sum of square deviation of the BOP determined properties from the real properties (obtained either from experiments or quantummechanical calculations) of the target structures does not lead to a good set of parameters for the complex BOP potential, regardless of which structures are included. Albe et al. ${ }^{48}$ discovered that for Tersoff potentials, the pairwise energy functions can be independently determined from the equilibrium target values of bond length, bond energy, and bulk modulus of a variety of structures without considering the angular terms. Once the pair functions are determined using this method, the angular function parameters can be optimized in a second step to best match additional properties. In Appendix C, we derive a similar two-step fitting method that is effective in high-quality BOP parameterization. Using this two-step method, the pairwise parameters $\phi_{0}, m, n$, and $n_{c}$ are first determined by fitting some properties of the nearest-neighbor structures using Eqs. (C3) and (C4). The remaining parameters are then determined in a second step by fitting other properties, including those of the non-nearest-neighbor structures.

\section{Constraints on parameters}

Appropriate target structures and fitting methods alone are not sufficient to create a physically sound BOP. Many parameters critically require valid bounds. It is not trivial to determine the bounds of all the parameters. The bounds of the parameters that we used do not necessarily represent the optimum choices but were obtained from a combination of physical intuition and extensive trial-and-error experimentations. These bounds are listed in Table V of Appendix D. Some key points are mentioned here. The ratio of the pair function parameters $\mathrm{m}$ to $\mathrm{n}$ equals the relative hardness $\frac{R^{\prime}(r)}{R(r)} / \frac{U^{\prime}(r)}{U(r)}$ characteristic of the shape of the energy curve, ${ }^{54}$ where $R(r)$ and $U(r)$ represent, respectively, the repulsive and attractive pair functions. For a hard-sphere potential, $m / n=\infty$, but for real materials, $m / n$ is normally around $2(m / n=2.2$ for carbon, $m / n=$ 1.8 for silicon, ${ }^{54}$ and the conventional Lennard-Jones ${ }^{62}$ and Morse $^{63}$ potentials both have $m / n=2$ ). Hence, $m / n$ is targeted at 2.0 with slight adjustment. In addition, the pair function parameters are constrained so that Eqs. (2)-(4) decay to small values near the cutoff distances even without multiplying them 
by the cutoff function. This ensures that the pair functions smoothly decay to zero when multiplied by the cutoff function. Finally, the parameters of the angular function are constrained so that Eq. (A5) is monotonic between $\theta=0^{\circ}$ and $\theta=180^{\circ}$.

\section{E. Fitting algorithm}

Symbolic computations were performed using Mathematica $^{64}$ to derive complicated expressions for the cohesive energies, pressure, and elastic constants of various structures. Four Mathematica built-in numerical optimization routines, namely a conjugate gradient method, ${ }^{65}$ the downhill simplex method of Nelder and Mead, ${ }^{66}$ a genetic algorithm, ${ }^{67}$ and biased random walk (simulated annealing), ${ }^{68}$ were all used to determine the parameters that minimize the mean-square difference between the target and predicted properties. These numerical routines require initial values of parameters that alter the difficulty level of the fitting. While we used a trial-and-error approach to get progressively better guesses of the initial parameters, future BOP development can use the CdTe parameters determined in this work or the GaAs parameters determined previously ${ }^{27}$ as good references for the initial parameters. After each fitting iteration, the four sets of parameters from the four optimization routines were tested for a larger collection of structures and vapor deposition simulations. If spurious low-energy structures existed (for example, a structure was found to have a cohesive energy lower than that of the ground-state phase), or vapor deposition simulations predicted an amorphous growth, the entire process was repeated with an appropriate adjustment of target structures and target properties. The iterations continued until one of the four optimization routines resulted in a satisfactory set of potential parameters. The parameterization was first conducted for elemental $\mathrm{Cd}$ and then for elemental Te. Finally, the parameters determined for $\mathrm{Cd}$ and Te were used in CdTe binary systems to determine the remaining parameters. The BOP potentials thus determined are listed in Tables I-IV of Appendix B for global, point-dependent, pair-dependent, and three-body-dependent parameters, respectively.

\section{EVALUATION OF THE POTENTIAL}

Using parallel MD code LAMMPS, ${ }^{28}$ extensive simulations were performed to evaluate and validate the BOP. In particular, geometries and energies of numerous small clusters; lattice constants, cohesive energies, and bulk moduli of a variety of lattice structures; and melting temperature, properties of common point defects (interstitials, vacancies, and antisites), and surface reconstructions of the lowest-energy zinc-blende CdTe compound, are all studied. The results obtained from both our BOP and $\mathrm{SW}^{30}$ and $\mathrm{TR}^{32}$ CdTe potentials in the literature are compared with those obtained from our highlevel DFT calculations (as described in Appendix E) and the published experiments. Finally, vapor deposition simulations are presented, which capture crystalline growth.

\section{A. Small-cluster properties}

To test BOP's transferability to different environments, bonding energies and geometries of a variety of $\mathrm{Cd}$, $\mathrm{Te}$, and CdTe clusters with different coordination numbers are studied.

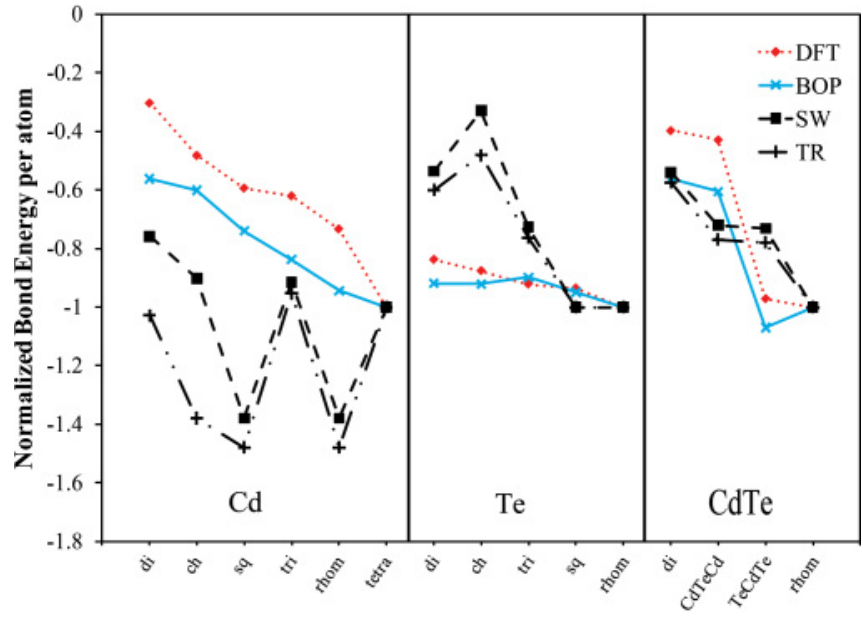

FIG. 2. (Color online) Normalized binding energies per atom for selected $\mathrm{Cd}$, Te, and CdTe clusters.

Relaxed structures are determined from molecular statics energy minimization simulations based upon a conjugate gradient method. ${ }^{69}$ Similar calculations are performed using the DFT method (see Appendix E). For numerical references, results of the bonding energies and geometries of a variety of small clusters are all tabulated in Tables VI through IX of Appendix F. It is important to note that the DFT method captures property trends but not necessarily absolute values. In addition, the structures that are likely to form in a dynamic simulation are really determined by the energy trends of different phases. As a result, we examine the energy trends of various clusters in Fig. 2. To focus on the trends as opposed to the magnitudes of the energies, the binding energies of different clusters shown in Fig. 2 are normalized by the magnitudes of the energy of the lowest-energy clusters determined in DFT calculations (tetra for $\mathrm{Cd}$, rhom for Te, and rhom for CdTe). It can be seen from Fig. 2 that BOP energy trends are significantly closer to the DFT values than those from the SW and TR potentials. In particular, BOP reproduces the DFT trends from the low- (large magnitude) to high-energy clusters for $\mathrm{Cd}$, namely tetra-rhom-tri-sq-ch-di. Except for a slightly underestimated magnitude of the energy for the Te trimer cluster, and a slightly overestimated magnitude of the energy for the TeCdTe trimer cluster, the BOP also gives correctly the energy trends for Te, namely rhom-sq-ch-di, and the energy trends for CdTe, namely rhom-CdTeCd-di.

Figure 2 compares the normalized energies. In terms of real values, Table VI indicates that for $\mathrm{Cd}$ clusters, BOP overpredicts the magnitude of the energies as compared with the DFT values, whereas for Te and CdTe clusters, the BOP magnitude of the energies agrees reasonably well with the DFT values (note that DFT and experimental values also differ). In addition to the binding energies, Tables VII through IX indicate that the bond lengths for the $\mathrm{Cd}$ clusters are underpredicted by $12-20 \%$. The bond length of Te and CdTe clusters also deviate from DFT values by $5-9 \%$ and $9-21 \%$, respectively. As discussed in Appendix C, the bond energy vs bond length relation of different (nearest-neighbor) structures always satisfy Eq. (C3) if $\phi$ and $\beta_{\sigma}$ are pair functions. Regardless of the parameters, the bond energy specified by 

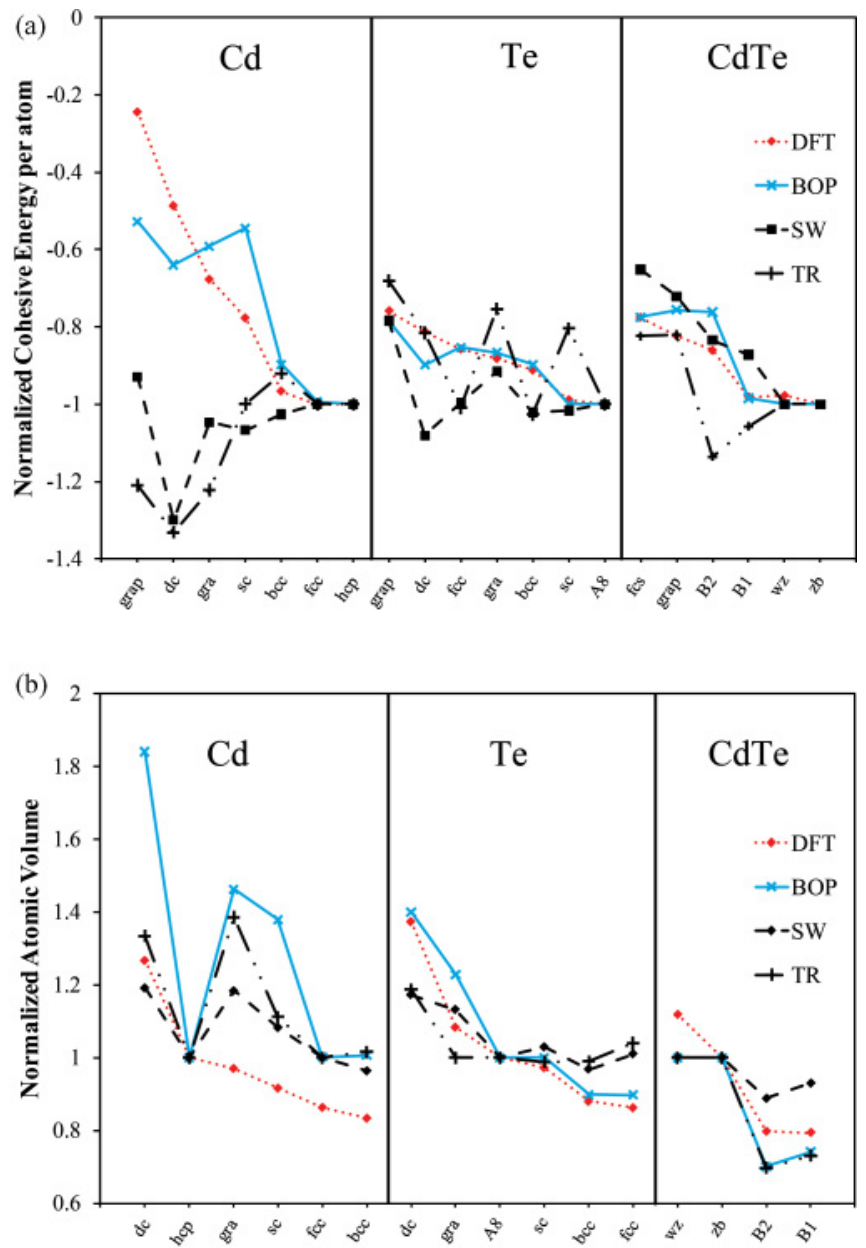

FIG. 3. (Color online) Normalized (a) cohesive energies and (b) atomic volumes of a variety of $\mathrm{Cd}, \mathrm{Te}$, and $\mathrm{CdTe}$ lattices.

Eq. (C3) is a monotonically increasing function of bond length. In contrast, the DFT bond energy vs bond length data is rather scattered. As a result, it is necessary that some differences exist between BOP and DFT. This observation was also observed in the previous work. ${ }^{27}$ We feel that while the improved energy trends are already a significant advantage of BOP over other potentials, further improvement of the BOP is possible by incorporating the environment dependence in the repulsive and bond integral functions, i.e., the $\phi$ and $\beta_{\sigma}$ in Eq. (C3) are no longer treated as pair functions.

\section{B. Bulk lattice structures}

Zero-pressure energy minimization simulations $\mathrm{s}^{70}$ are performed to determine the relaxed structures and properties of different bulk lattices using our BOP, $\mathrm{SW},{ }^{30}$ and $\mathrm{TR}^{32}$ potentials, as well as the DFT calculations. A variety of $\mathrm{Cd}$, $\mathrm{Te}$, and CdTe lattices with coordination numbers between 4 and 12 are considered. Again for numerical references, the lattice constants and cohesive energies obtained from different models and the available experimental data are tabulated in Tables X through XII of Appendix F for Cd, Te, and CdTe lattices, respectively. Here we compare cohesive energies and atomic volumes (can be related to lattice constants) obtained from different models and available experiments ${ }^{71,72}$ in Figs. 3(a) and 3(b), respectively. To again focus on trends, energies and volumes shown in Fig. 3 are normalized by the magnitude of the energy and volume of the experimentally observed equilibrium structures for each model.

Experiments indicated that equilibrium phases for $\mathrm{Cd}, \mathrm{Te}$, and $\mathrm{CdTe}$ are hcp, A8, and $\mathrm{zb}$ with cohesive energies of $-1.133 \mathrm{eV} /$ atom, $-2.168 \mathrm{eV} /$ atom, and $-2.178 \mathrm{eV} /$ atom, respectively. ${ }^{71,72}$ Figure 3(a) indicates that compared with the SW and TR potentials, BOP calculates energy trends significantly closer to those of the DFT method. Most importantly, the BOP correctly captures the Cd-hcp, Te-A8, and CdTe-zb as the lowest energy phases with an exact match of their cohesive energies (refer to Tables X through XII). In sharp contrast, the lowest energy phases are calculated to be $\mathrm{Cd}-\mathrm{dc}, \mathrm{Te}-\mathrm{dc}$, and CdTe-zb by the SW potential ${ }^{30}$ and Cd-dc, Te-bcc, and CdTe-B2 by the TR potential. ${ }^{32}$ In fact, the SW potential has the only correct lowest-energy lattice of CdTe-zb. These results indicate that the TR potential cannot be used to study any of the equilibrium $\mathrm{Cd}, \mathrm{Te}$, or $\mathrm{CdTe}$ phases. Although the SW potential seems to work for the equilibrium CdTe phase, the results may be inaccurate as the potential does not transfer to the $\mathrm{Cd}$ and $\mathrm{Te}$ (and hence the defective) regimes. As a result, our CdTe BOP significantly improves over other widely used potentials on energy trends of different configurations, leading to a better description of defects in CdTe crystals.

Based on lattice constants, ${ }^{72}$ the experimental atomic volumes of the equilibrium Cd-hcp, Te-A8, and CdTe-zb phases are $21.46 \AA^{3}$ /atom, $33.76 \AA^{3}$ /atom, and $33.98 \AA^{3}$ /atom, respectively. Figure $3(\mathrm{~b})$ indicates that compared with the other two literature potentials, BOP significantly improves the calculated atomic volume trends of different $\mathrm{Te}$ and $\mathrm{CdTe}$ phases. The largest deviation of the volume trends between BOP and DFT occurs for the Cd phases. The only reason attributed to this discrepancy is a large drop of the atomic volume of the Cd-hcp phase. Experimentally, the Cd-hcp has a significantly larger lattice constant ratio of $\mathrm{c} / \mathrm{a}=1.885,{ }^{72}$ as compared with the ideal ratio of 1.633 . With extensive efforts, we discovered that BOP could not fit a c/a ratio significantly above the ideal ratio. As a result, we could not fit a bigger atomic volume for the Cd-hcp phase, while ensuring other property trends. Note that this issue is not specific to BOP; it also occurs for other potentials. ${ }^{44}$ In fact, one previous method to fit c/a ratio is to modify a SW potential so that the pair functions give two energy minima to accommodate two lattice constants $a$ and $c{ }^{73}$ Such an approach is obviously not desired. Again, it should be recognized that the atomic volume trends are constrained by Eq. (C3) when $\phi$ and $\beta_{\sigma}$ are treated as pair functions. Further improvement can be achieved when $\phi$ and $\beta_{\sigma}$ are not approximated as pair functions. Nonetheless, the overall improvement of our BOP on the atomic volume trends over the other potentials, Fig. 3(b), enables more accurate description of strain energies when the system undergoes phase transformation.

To further examine the fidelity of the BOP for CdTe simulations, the single-crystal elastic constants of the $\mathrm{CdTe}-\mathrm{zb}$ phase are calculated and compared for different models and literature experimental ${ }^{74} / \mathrm{DFT}^{75}$ data in Table XIII of Appendix F. It can be seen that the BOP captures the elastic constants of the CdTe-zb phase remarkably well, which significantly improves 


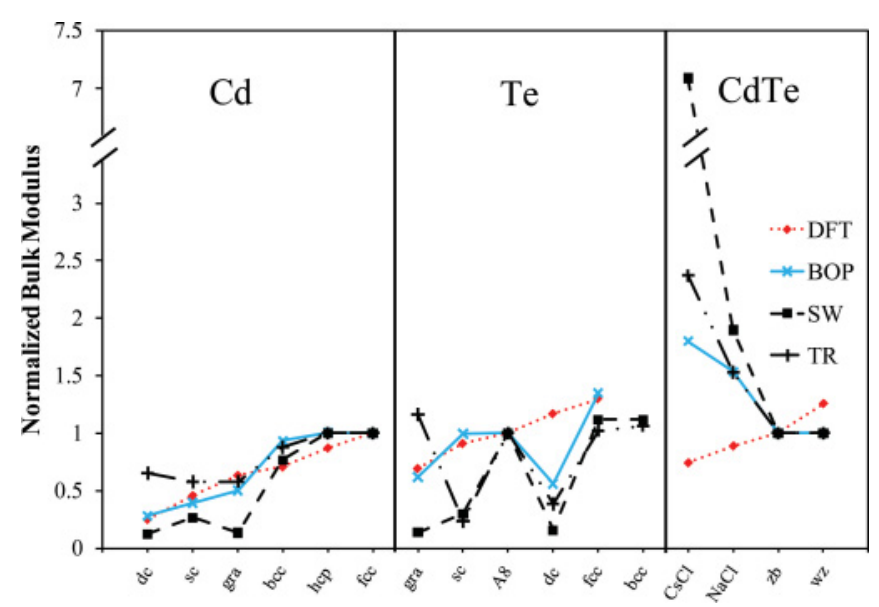

FIG. 4. (Color online) Normalized bulk moduli of a variety of $\mathrm{Cd}, \mathrm{Te}$, and CdTe phases.

over the SW potential (the only other potential having the correct lowest-energy CdTe phase). Note that these elastic constants are not fitted, and their good values are indicative of the transferability of the BOP model.

The bulk moduli of a variety of $\mathrm{Cd}, \mathrm{Te}$, and CdTe phases are also calculated using different methods, and the normalized results are compared in Fig. 4. It can be seen from Fig. 4 that BOP does a reasonable job of capturing the bulk modulus trends of various phases. In particular, the bulk modulus trends predicted by the BOP are significantly closer to those of the DFT method, and BOP does not suffer from the problem of an abnormally high bulk modulus of the CdTe-B2 phase as shown by the SW potential.

In general, the BOP accurately captures the most important energy trends of a variety of $\mathrm{Cd}$, $\mathrm{Te}$, and $\mathrm{CdTe}$ phases. It also significantly improves the prediction of trends for atomic volumes and elastic constants of different $\mathrm{Cd}, \mathrm{Te}$, and $\mathrm{CdTe}$ phases over the other available potentials.

\section{Melting temperature}

Melting temperature simulations test a large number of thermally activated configurations and have implications on modeling thermodynamic properties. Thus, we calculate the melting temperature of the $\mathrm{CdTe}-\mathrm{zb}$ phase. We adopt the MD technique proposed by Morris et al. ${ }^{76}$ for which one slab of CdTe-zb crystal and one slab of liquid CdTe reach an equilibrium temperature together. The simulations employ periodic boundary conditions in all three coordinate directions. We begin with a single-crystalline $\mathrm{CdTe}$-zb crystal containing 7200 atoms constructed using the $0-\mathrm{K}$ lattice parameter. The crystalline half of the block contains 3600 atoms with positions held fixed, while increasing the temperature of the other 3600 atoms to $2700 \mathrm{~K}$ over a $0.1-\mathrm{ns}$ period. The pressure is held at 1 atm under the NPT (constant number of atoms, pressure, and temperature) conditions using a Nosè-Hoover thermostat/barostat, ${ }^{77,78}$ with a temperature damping parameter of $10.0 \mathrm{ps}$ and a pressure damping parameter of $5.0 \mathrm{ps}$. The constant pressure condition ensures that the sample dimension could relax, thereby removing any unrealistic stresses in the melt. After reaching a constant temperature for $0.05 \mathrm{~ns}$, the fixed crystal is released. Isenthalpic NPH (constant number of atoms, pressure, and enthalpy) dynamics is then performed for $0.2 \mathrm{~ns}$ to bring the two slabs into thermal equilibrium where the system temperature is well converged. Another 0.05-ns simulation is conducted, and the melting temperature is calculated as the average temperature in the final 0.05 -ns period.

The CdTe-zb melting temperatures calculated by different methods are compared in Table XIV of Appendix F along with the available experimental value. ${ }^{79}$ It can be seen that the TR potential significantly underpredicts the melting temperature as the CdTe-zb phase is not even the equilibrium phase with the TR potential. With only the CdTe-zb phase considered, the SW potential parameterization explicitly fits the melting temperature by adjusting the cutoff radius. ${ }^{30}$ As a result, it is not surprising that the SW potential calculates the melting temperature exactly. Without an explicit fitting, the BOP prediction of the melting temperature comes remarkably close to the experimental value. BOP could possibly approach a closer value to the experimental melting temperature by adjusting the cutoff radius. With so many phases considered, this refinement is not trivial and is not pursued in the current work.

\section{Point defects}

In addition to examining different clusters and lattices, defect properties are also studied to further test the BOP potential. The primary native defects observed in $\mathrm{CdTe}$ compounds are $\mathrm{Cd}$ interstitials under the $\mathrm{Cd}$-rich condition and $\mathrm{Cd}$ vacancies, Te interstitials, and Te antisites under the Te-rich conditions. ${ }^{80,81}$ Various types of defects can be easily introduced in the computational crystal. The stoichiometry of the system containing the defects, however, does not necessarily equal the stoichiometry of the perfect crystal. Following the methodology of Zhang and Northrup, ${ }^{27,82,83}$ the defect energy $\Gamma$ is calculated as a function of the chemical potential difference $\Delta \mu$ as

$$
\Gamma=E_{D}^{\prime}-0.5\left(n_{\mathrm{Cd}}-n_{\mathrm{Te}}\right) \cdot \Delta \mu,
$$

where $n_{\mathrm{Cd}}$ and $n_{\mathrm{Te}}$ are numbers of $\mathrm{Cd}$ and Te atoms in the defective system, $\Delta \mu$ is the chemical potential difference characteristic of the environment, and $E_{D}^{\prime}$ is an intrinsic defect energy at stoichiometric condition. $\Delta \mu$ is expressed as

$$
\Delta \mu=\left(\mu_{\mathrm{Cd}}-\mu_{\mathrm{Cd}}^{\text {bulk }}\right)-\left(\mu_{\mathrm{Te}}-\mu_{\mathrm{Te}}^{\text {bulk }}\right),
$$

where $\mu_{\mathrm{Cd}}$ and $\mu_{\mathrm{Te}}$ are the chemical potentials of $\mathrm{Cd}$ and $\mathrm{Te}$ in the CdTe compound and $\mu_{\mathrm{Cd}}^{\text {bulk }}$ and $\mu_{\mathrm{Te}}^{\text {bulk }}$ are the chemical potentials for the lowest-energy $\mathrm{Cd}$ and $\mathrm{Te}$ phases. In our work, all chemical potentials are approximated as cohesive energies per atom unit. Under this approximation, $\Delta \mu$ satisfies the condition $-\Delta H_{f}<\Delta \mu<\Delta H_{f}$, where $\Delta H_{f}$ is heat of mixing. ${ }^{83}$ In general, $\Delta \mu=0, \Delta \mu>0$, and $\Delta \mu<0$ mean stoichiometric, $\mathrm{Cd}$-rich, and Te-rich conditions.

The intrinsic defect energy can be calculated as

$$
\begin{aligned}
E_{D}^{\prime}= & E_{D}-0.5\left(n_{\mathrm{Cd}}+n_{\mathrm{Te}}\right) \cdot \mu_{\mathrm{CdTe}}^{\text {bulk }}-0.5\left(n_{\mathrm{Cd}}-n_{\mathrm{Te}}\right) \\
& \cdot\left(\mu_{\mathrm{Cd}}^{\mathrm{bulk}}-\mu_{\mathrm{Te}}^{\mathrm{bulk}}\right),
\end{aligned}
$$

where $E_{D}$ is the total energy of the system containing the defect, and $\mu_{\mathrm{CdTe}}^{\text {bulk }}$ is the chemical potential of the lowest-energy CdTe phase. Under the equilibrium condition $\Delta \mu=0$ and 


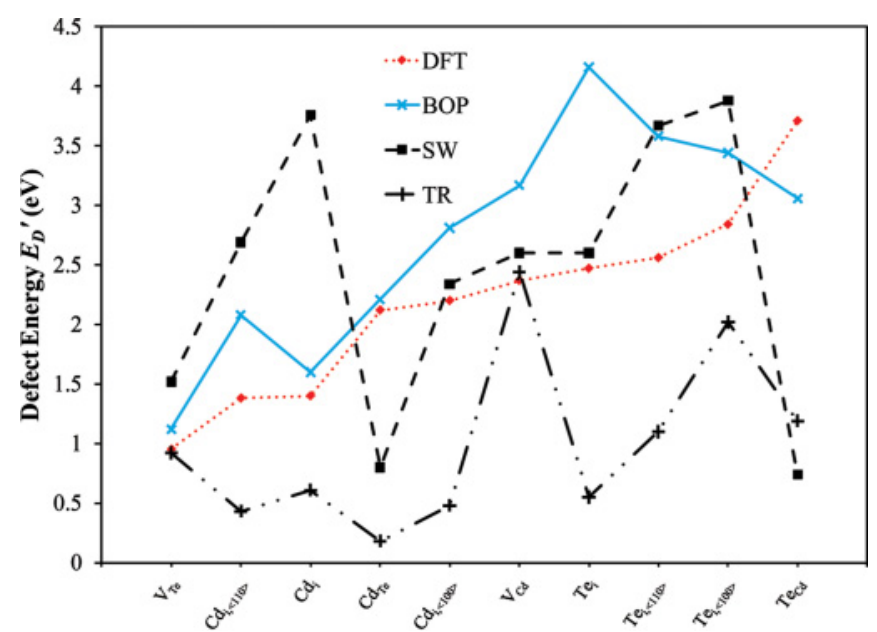

FIG. 5. (Color online) Various defect energies of the CdTe-zb phase.

$\Gamma=E_{D}^{\prime}$. Under the Cd-rich or Te-rich condition, $\Delta \mu \neq 0$ and thus $\Gamma \neq E_{D}^{\prime}$.

The defects considered here include $\mathrm{Cd}$ vacancy $\left(\mathrm{V}_{\mathrm{Cd}}\right)$, Te vacancy $\left(\mathrm{V}_{\mathrm{Te}}\right), \mathrm{Cd}$ at $\mathrm{Te}$ antisite $\left(\mathrm{Cd}_{\mathrm{Te}}\right)$, Te at $\mathrm{Cd}$ antisite $\left(\mathrm{Te}_{\mathrm{Cd}}\right), \mathrm{Cd}$ interstitial surrounded by the Te tetrahedron shell $\left(\mathrm{Cd}_{i}\right)$, and $\mathrm{Te}$ interstitial surrounded by the $\mathrm{Cd}$ tetrahedron shell $\left(\mathrm{Te}_{i}\right)$. In addition, $\langle 110\rangle$ and $\langle 100\rangle$ dumbbell interstitials ${ }^{84}$ are also considered. The dumbbell interstitials are formed by splitting an onsite $\mathrm{Cd}$ or Te atom into two atoms and separating them with their bond aligned along either the $\langle 100\rangle$ or $\langle 110\rangle$ direction. $^{84,85}$

Energy minimization simulations are performed to calculate the total energies of the CdTe-zb systems (with about 512 atoms) containing the corresponding defects, $E_{D}$. The intrinsic defect energies are then calculated using Eq. (11). The results obtained from various models are numerically tabulated in Table XV of Appendix F and are reproduced in Fig. 5 for a more convenient examination.

Figure 5 indicates that the defect energy trends predicted by the BOP are significantly closer to those by the DFT method than the other potentials. Most importantly, the BOP captures correctly the Te vacancy as the lowest energy defect with a comparable defect energy with the DFT value. Contrarily, the SW potential predicts the $\mathrm{Cd}_{\mathrm{Te}}$ antisite defect to have the lowest energy. Worse than that, the TR potential predicts at least five other defects to have a lower energy than Te vacancy. Remarkably, the BOP also captures well the energy trends and magnitude of energies of $\mathrm{Cd}_{i,\langle 110\rangle}, \mathrm{Cd}_{i}$, and $\mathrm{Cd}_{\mathrm{Te}}$. These three additional defects, along with $\mathrm{V}_{\mathrm{Te}}$, are important as they have the four lowest energies in the DFT calculations. BOP, therefore, can be used to accurately study defect problems.

\section{E. CdTe surfaces}

Simulations of mechanical processes (such as fracture) and growth processes (such as vapor deposition), sample surface configurations. To evaluate the transferability of our BOP to these scenarios, the $(010) \mathrm{CdTe}-\mathrm{zb}$ surface is studied. The (010) surface of the CdTe-zb crystal has exhibited a variety of surface reconstructions depending on the environment. ${ }^{86-88}$ Some possible surface reconstructions, as reported by Gundel

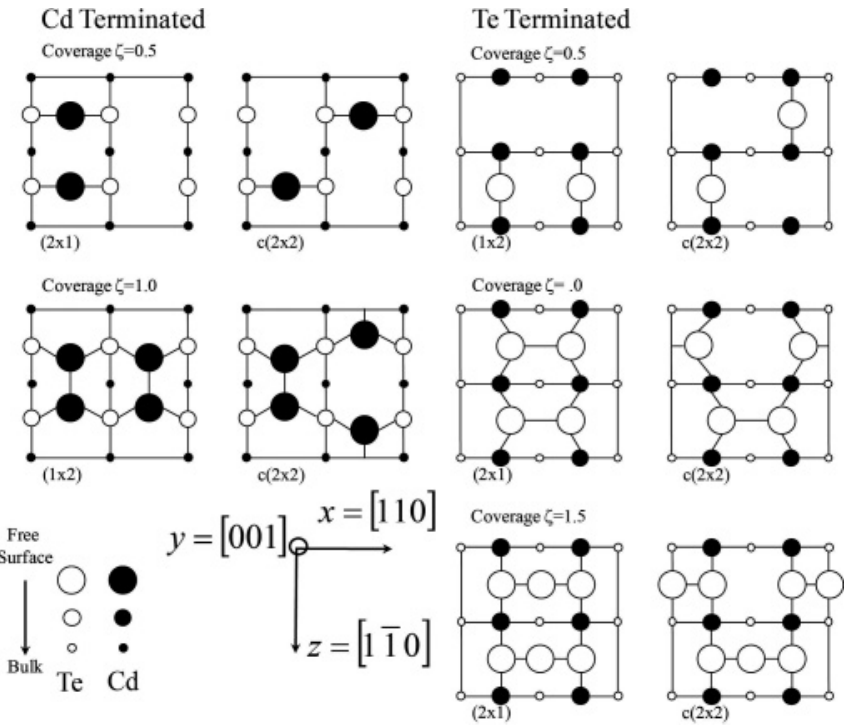

FIG. 6. Possible (010) CdTe-zb surface reconstructions.

et $a l .{ }^{89}$ are shown in Fig. 6. Using DFT simulations, Gundel et al. ${ }^{89}$ predicted that for Te-rich environments, the Te $(2 \times 1)$ reconstruction (Te coverage of $\xi=1.0$ ) is favorable, whereas for Cd-rich environments, the $\mathrm{Cd} \mathrm{c}(2 \times 2)(\mathrm{Te}$ coverage $\xi=0.5)$ is favorable. Gundel's calculations reproduce the experimental observation of $\mathrm{Te}(2 \times 1)$ reconstructions in Terich environments ${ }^{90}$ and $\mathrm{Cd} \mathrm{c}(2 \times 2)$ surface reconstructions in Cd-rich environments. ${ }^{86}$

Based upon Eqs. (9) and (11), we calculate the energies of all the $10 \mathrm{CdTe}-\mathrm{zb}$ (010) surface reconstructions shown in Fig. 6 as a function of the chemical potential difference, Eq. (10). In the MD simulations performed here, $\Delta \mu$ is left as an independent variable representing transition from Te-rich to Cd-rich environments. The computational cell used in each simulation contains a block of zb CdTe crystal with $\sim 2300-2500$ atoms. Periodic boundary conditions are used in the $x$ and $z$ directions and two parallel free surfaces (with the same reconstructions) are created in the $\pm y$ directions. The two surfaces are not perfectly symmetric as one of the free surfaces is rotated $90^{\circ}$ relative to the opposite surface. The calculated surface energies are summarized in Figs. 7(a) and 7(b) for the BOP potential and DFT, respectively. Figure 7(a) indicates that within the possible range of chemical potential difference between $-\Delta H_{f}$ and $\Delta H_{f}$, the preferred surface for the BOP potential is Te $(2 \times 1)$ with coverage $\xi=1.0$ in the Te-rich environments $\left(\Delta \mu\right.$ near the $-\Delta H_{f}$ end), in good agreement with Gundel's calculations. ${ }^{89}$ In addition, Fig. 7(a) shows that in the Cd-rich environments $(\Delta \mu$ near the $\Delta H_{f}$ end $)$, the preferred surface is $\mathrm{Cd}(1 \times 1)$ with coverage $\xi=1.0$ (this surface can also be considered as bulk terminated). Fig. Fig. 7(b) indicates the preferred surfaces predicted by our DFT are the $\mathrm{Cd}(2 \times 1)$ with coverage $\xi$ $=0.5$ and the $\mathrm{Cd} \mathrm{c}(2 \times 2)$ with coverage $\xi=0.5$ in the Te-rich environments and $\mathrm{Cd}(1 \times 2)$ with coverage $\xi=1.0$ in the $\mathrm{Cd}$-rich environments. Interestingly, BOP does not predict dimerization for the Cd-terminated surfaces with coverage $\xi=1.0$. This is supported by experiments ${ }^{86}$ but has not been predicted by other potentials or DFT. ${ }^{35}$ 

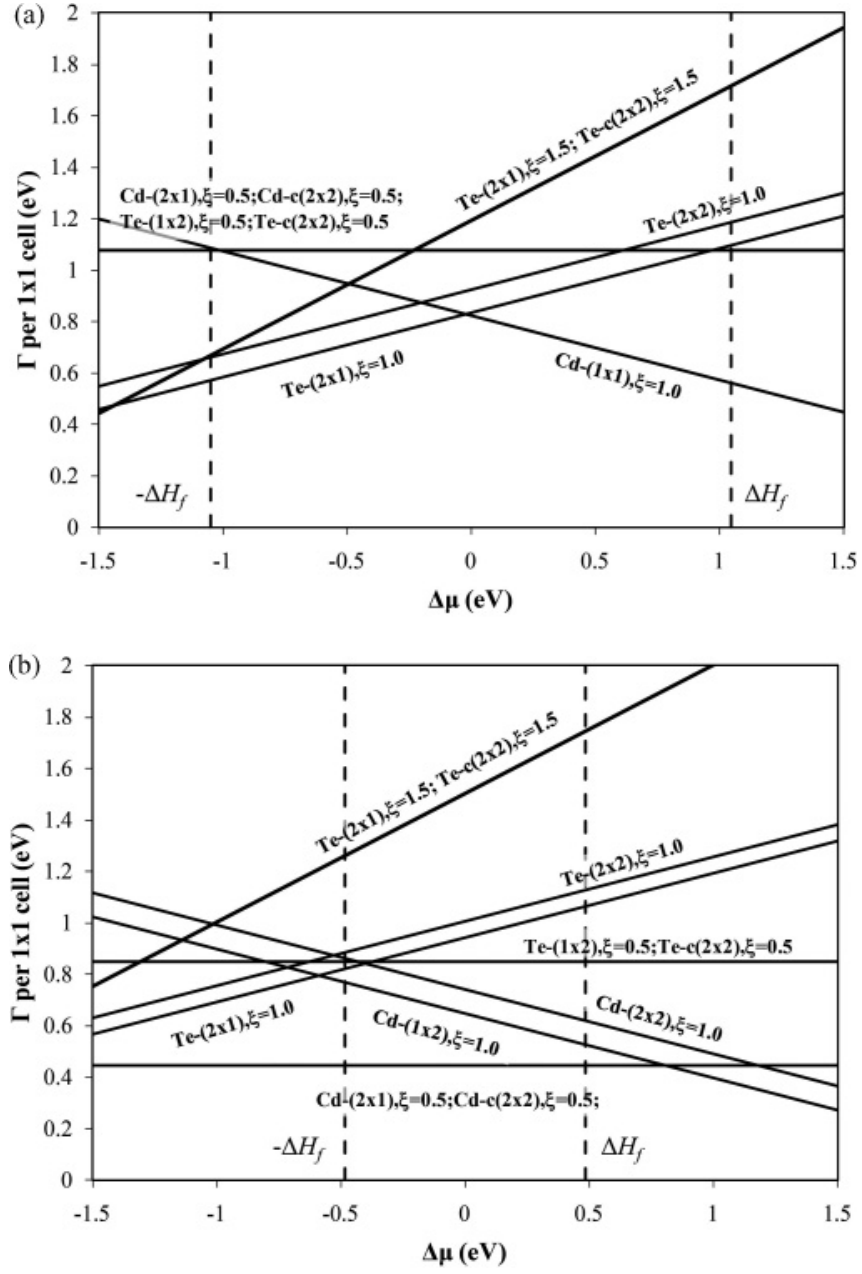

FIG. 7. (010) CdTe surface energy phase diagrams predicted by (a) BOP and (b) DFT.

\section{F. Vapor deposition simulations}

SW potentials do not capture well the property trends of different phases ${ }^{35}$ but can be easily parameterized to generate crystalline growth. ${ }^{91-93}$ Although Tersoff potentials more accurately exhibit the property trends, they are difficult to parameterize. As a result, Tersoff potentials in the literature are often found to predict amorphous growth. ${ }^{32,35,45}$ BOP fundamentally improves on the prediction of property trends over Tersoff potentials. Therefore, BOP can greatly improve the way atomistic simulations are performed for semiconductor systems if it is well parameterized to capture both property trends and crystalline growth. To evaluate BOP behavior on growth simulations, we perform MDs simulations of vapor deposition of Cd-hcp, Te-A8, and CdTe-zb growth. Our computational systems employ periodic boundary conditions in the $x$ and $z$ directions and a free boundary condition in the $y$ direction. The growth occurs in the $+y$ direction, and a constant zero pressure is maintained during simulations to relax the system dimensions.

For Cd growth, an initial substrate of an hep crystal containing $1536 \mathrm{Cd}$ atoms with $24(2 \overline{1} \overline{1} 0)$ layers in the $x$ direction, 9 (0002) layers in the $y$ direction, and 16 (0110) layers in the $z$ direction is used, where layers refer to crystallographic planes so that $\mathrm{n}(000 \mathrm{n})$ layer is equivalent to

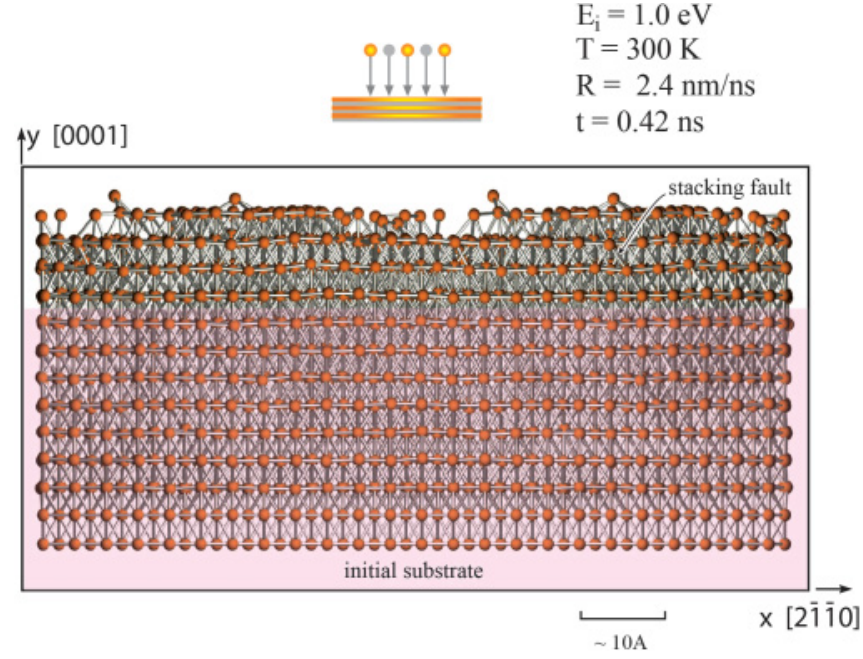

FIG. 8. (Color online) Predicted growth of hcp Cd in the [0001] direction.

one (0001) layers, etc. The substrate temperature is set at $\mathrm{T}=$ $300 \mathrm{~K}$ by assigning velocities to atoms according to the Boltzmann distribution. During simulations, the bottom (-y) two (0002) layers are held fixed to prevent crystal shift upon adatom impact on the top surface. The next three (0002) layers are isothermally controlled at the substrate temperature. This leaves the top four layers free, where the motion of atoms is solely determined by Newton's law. Growth is simulated by injecting $\mathrm{Cd}$ adatoms from random locations far above the surface. All adatoms have an initial far-field incident kinetic energy $E_{i}=1.0 \mathrm{eV}$ and an incident angle $\theta=0^{\circ}$ (i.e., the moving direction is perpendicular to the surface). The adatom injection frequency is chosen to give a deposition rate of $R=2.4 \mathrm{~nm} / \mathrm{ns}$. This deposition rate is much higher than experimental values. However, by maintaining the substrate at an elevated temperature, adatoms have a significant energy to locate low energy wells on the surface even within the short-time constraint imposed by the high deposition rate. To approximately maintain a constant thickness of the free surface region, the isothermal region expands upward during simulations. Because surface roughness might develop, the isothermal region expands at about $80 \%$ of the surface growth rate so that the upper boundary of the isothermal region never exceeds the surface even at the valley locations. The resulting configuration obtained after 0.42-ns deposition is shown in Fig. 8. Note that in the figure, the $x$ dimension of the system has been extended using periodic boundary conditions, and the original substrate is shaded in pink. It can be seen that except for the formation of stacking fault on the (0001) surface, our BOP correctly captures the crystalline growth of the $\mathrm{Cd}$.

For Te growth, an initial substrate of an A8 crystal containing $1440 \mathrm{Te}$ atoms with 20 (21) 10$)$ layers in the $x$ direction, 12 (0003) layers in the $y$ direction, and $12(0 \overline{1} 10)$ layers in the $z$ direction is used. The bottom three (0003) layers are fixed, the next three (0003) layers are isothermally controlled, and the top four (0003) layers are free. Growth is simulated at a substrate temperature of $T=300 \mathrm{~K}$, an adatom incident energy $E_{i}=0.1 \mathrm{eV}$, an incident angle $\theta=0^{\circ}$, and a deposition rate $R=3.5 \mathrm{~nm} / \mathrm{ns}$. Again, the isothermal region expands at about $80 \%$ of the growth rate. The configuration 

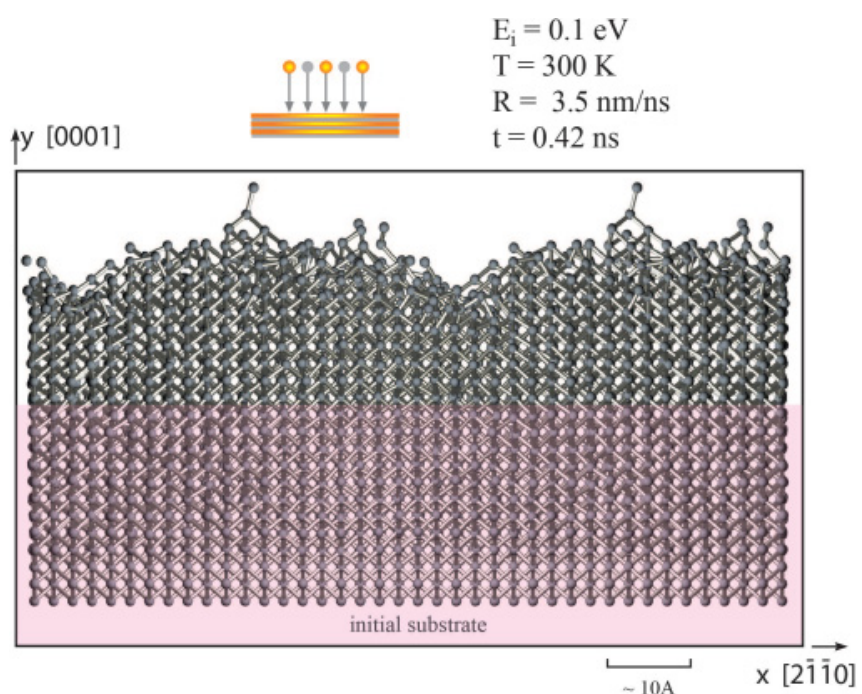

FIG. 9. (Color online) Predicted growth of A8 Te in the [0001] direction.

obtained after 0.42-ns deposition is shown in Fig. 9. Figure 9 further confirms that our BOP correctly captures the crystalline growth of the Te-A8 phase.

For CdTe growth, an initial substrate of a zb crystal containing $216 \mathrm{Cd}$ atoms and $216 \mathrm{Te}$ atoms with six (101)

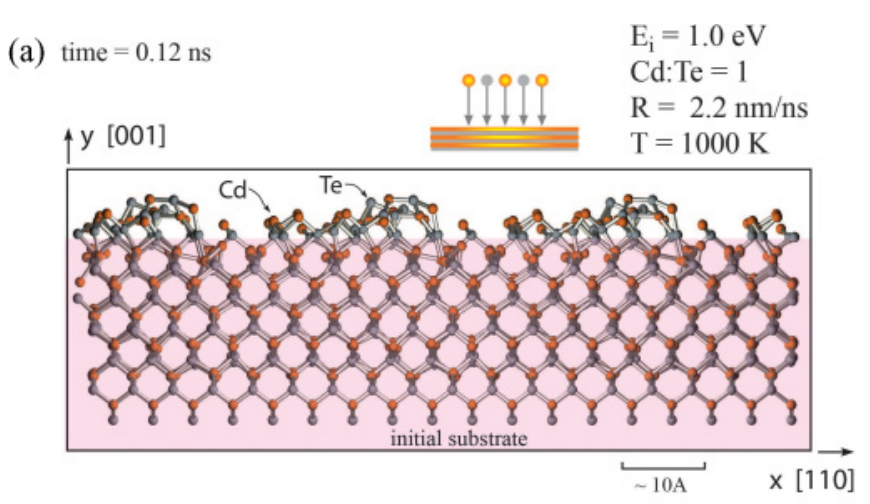

(b) time $=1.20 \mathrm{~ns}$

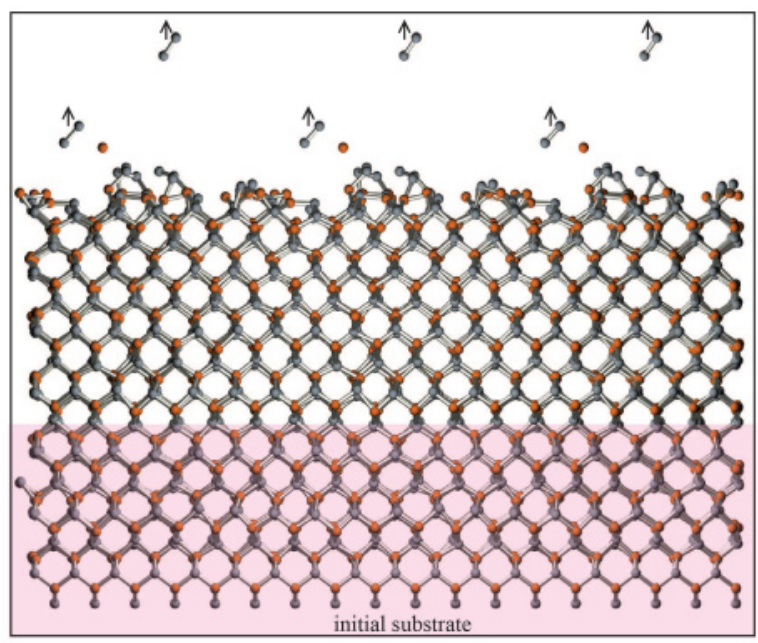

FIG. 10. (Color online) Predicted growth of zb CdTe in the [010] direction. (a) time $=0.12 \mathrm{~ns}$; and (b) time $=1.20 \mathrm{~ns}$. layers in the $x$ direction, 12 (040) layers in the $y$ direction, and six (101) layers in the $z$ direction is used. The top $y$ surface is terminated by Cd initially. During simulations, the bottom three (040) layers are fixed. To mimic the molecular beam epitaxy (MBE) growth condition commonly used for semiconductor growth, the isothermal region is expanded to include all atoms above the fixed region. To capture the adatom incident energy effects, however, the newly added adatoms are not isothermally controlled until they are fully incorporated into the film and their initial kinetic and potential (latent heat release) energies are fully dissipated. Growth is simulated at a substrate temperature of $T=1000 \mathrm{~K}$, an adatom incident energy $E_{i}=1.0 \mathrm{eV}$, an incident angle $\theta=0^{\circ}$, and a deposition rate $R=2.2 \mathrm{~nm} / \mathrm{ns}$. During simulation, $\mathrm{Cd}$ and Te adatoms from random locations far above the surface are continuously injected into the system. The injection of $\mathrm{Cd}$ and $\mathrm{Te}$ species is random but over time gives an average 1:1 stoichiometric ratio. The system configurations obtained at $0.12-$ and 1.2 -ns deposition times are shown, respectively, in Figs. 10(a) and 10(b). Figures 10(a) and 10(b) again confirm that our BOP correctly captures the crystalline growth of the $\mathrm{zb} \mathrm{CdTe}$. Interestingly, Fig. 10(b) shows that excess Te atoms on the surface evaporated in the form of molecular $\mathrm{Te}_{2}$ dimer.

\section{CONCLUSIONS}

We have developed a bond-order potential for the CdTe binary system. This potential is a significant improvement over previous formulations. Unlike other potentials currently available, this BOP simultaneously meets three criteria: (a) it is derived directly from quantum-mechanical theories and has a one-on-one correspondence with the tight-binding model; (b) it accurately captures property trends of many configurations; and (c) it results in crystalline growth in vapor deposition simulations. High-quality parameterization was achieved by considering a large number of target structures with coordinations ranging from 2 to 12; applying the two-step fitting approach; setting physically valid bounds for all parameters; using different minimization schemes and good initial guesses of parameters; and iterating the parameterization with crystalline growth simulation as part of

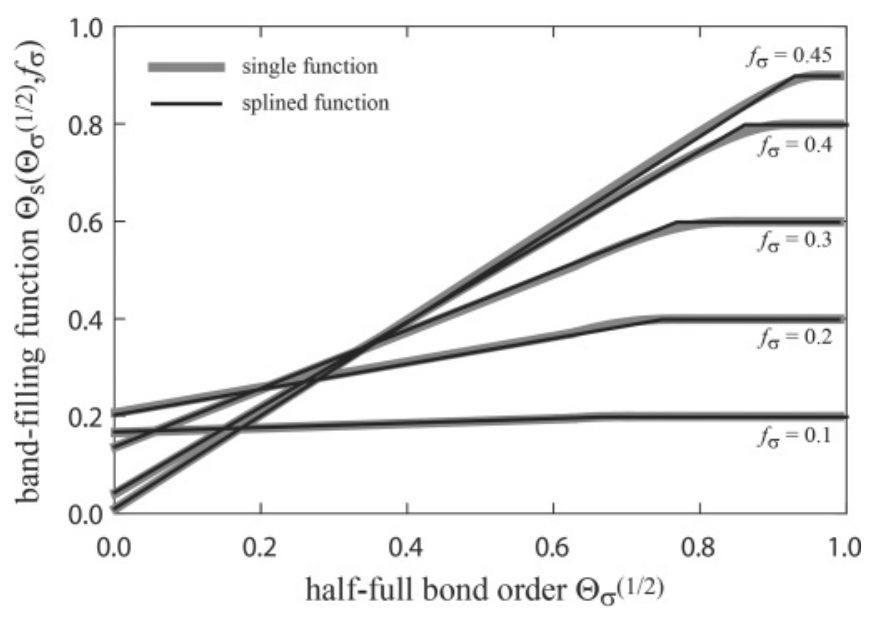

FIG. 11. $\Theta_{s, i j}\left(\Theta_{\sigma, i j}^{(1 / 2)}, f_{\sigma, i j}\right)$ as a function of $\Theta_{\sigma, i j}^{(1 / 2)}$ at different $f_{\sigma, i j}$ values calculated using the splined function ${ }^{27,42}$ (thick gray line) and Eq. (14) (thin black line). 
the validation tests. This potential captures well the property trends of clusters and lattices, melting, defects, and surfaces, and reproduces crystalline growth during vapor deposition simulations. We are confident that this potential will offer new opportunities for studying atomic-scale defects in CdTe components as well as the roles they play in the formation of larger defects. In addition, we expect that the emergence of such BOP potentials will enable empirical MD simulations of semiconductors to achieve a new fidelity level comparable to quantum-mechanical methods.

\section{ACKNOWLEDGMENTS}

We would like to thank David G. Pettifor for his guidance and comments throughout the development of this potential. This work is supported by the DOE/NNSA Office of Nonproliferation Research and Development, Proliferation Detection Program, Advanced Materials Portfolio. Sandia National Laboratories is a multiprogram laboratory managed and operated by Sandia Corporation, a wholly owned subsidiary of Lockheed Martin Corporation, for the US Department of Energy's National Nuclear Security Administration under Contract No. DE-AC04-94AL85000.

\section{APPENDIX A: ADDITIONAL FUNCTIONS FOR THE BOND-ORDER POTENTIAL}

The cutoff function used in Eqs. (2)-(4) is expressed as

$$
f_{c, i j}\left(r_{i j}\right)= \begin{cases}\frac{\exp \left(-\alpha_{i j} \cdot r_{i j} r_{i j}\right)-\exp \left(-\alpha_{i j} \cdot r_{\mathrm{cut}, i j}^{\gamma_{i j}}\right)}{\exp \left(-\alpha_{i j} \cdot r_{1, i j}\right)-\exp \left(-\alpha_{i j} \cdot r_{\mathrm{cut}, i j}\right)}, & r_{i j}<r_{\mathrm{cut}, i j} \\ 0, & r_{i j} \geqslant r_{\mathrm{cut}, i j}\end{cases}
$$

where $r_{1, i j}, r_{\text {cut }, i j}, \alpha_{i j}$, and ${ }_{i j}$ are pairwise parameters. $\alpha_{i j}$ and $\gamma_{i j}$ are defined from $\exp \left(-\alpha r_{\text {cut }, i j}^{\gamma}\right)=0.01$ and

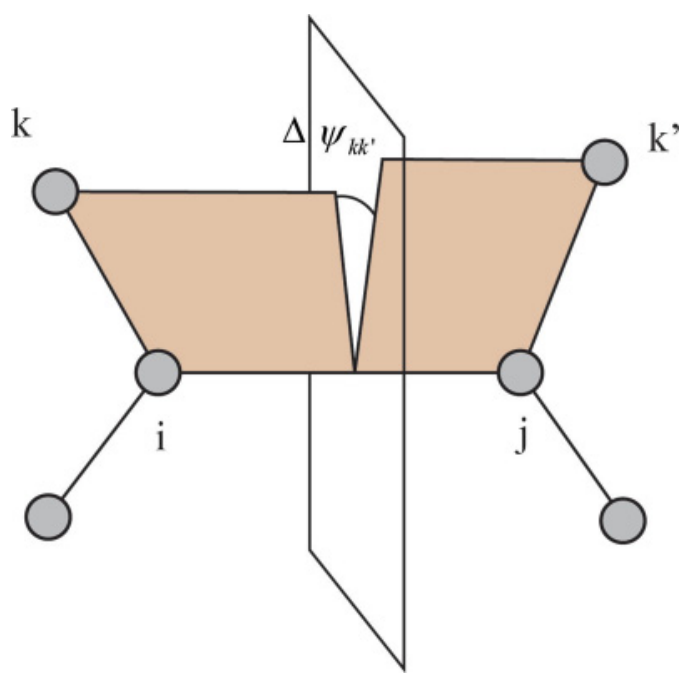

FIG. 12. (Color online) Dihedral angle $\Delta \psi_{k k^{\prime}}$ for the four-body involving the $k, i, j, k^{\prime}$ atoms around the $i j$ bond.
TABLE I. Global BOP parameters for CdTe.

\begin{tabular}{llc}
\hline \hline Symbol & \multicolumn{1}{c}{ Term } & - \\
\hline$\varsigma_{1}$ & Small number in Eq. (6) & 0.00001 \\
$\varsigma_{2}$ & Small number in Eq. (7) & 0.00001 \\
$\varsigma_{3}$ & Small number in Eq. (8) & 0.00100 \\
$\varsigma_{4}$ & Small number in Eq. (8) & 0.00001 \\
\hline \hline
\end{tabular}

$$
\begin{gathered}
\exp \left(-\alpha r_{1, i j}^{\gamma}\right)=0.99 \text {, giving } \\
\gamma_{i j}=\frac{\ln [\ln (0.99) / \ln (0.01)]}{\ln \left(r_{1, i j} / r_{\text {cut }, i j}\right)} \\
\alpha_{i j}=-\frac{\ln (0.99)}{\left(r_{1, i j}\right)^{\gamma_{i j}}} .
\end{gathered}
$$

Hence Eq. (A1) uses only two parameters $r_{1, i j}$ and $r_{\text {cut }, i j}$, with the latter being the cutoff distance of the functions. It can be seen that given Eqs. (A2) and (A3), the cutoff function in Eq. (A1) approximately equals unity when $r_{i j}$ is smaller than $r_{1, i j}$ and equals zero when $r_{i j}$ is larger than $r_{\text {cut }, i j}$. Multiplying the potential pair functions with Eq. (A1) therefore enables a smooth cutoff of the functions. A smooth decay near the cutoff is important under surfaces and liquid environments where frequent sampling of this region of the potential occurs. Additionally, smooth functions increase the stability of the numerical integrals at discrete time steps. ${ }^{94,95}$ The importance of the smooth cutoff has also been nicely illustrated when the GaN Tersoff potential ${ }^{47}$ is used to calculate $\mathrm{GaN}$ thermal conductivity. ${ }^{96}$ It is known that with the cutoff procedure used in the Tersoff potentials, ${ }^{34}$ only values and first derivatives of the pairwise energy functions are continuous, and the second derivatives are discontinuous. It was demonstrated ${ }^{96}$ that this can result in a significant underestimation of the thermal conductivity, and can also cause a large energy drift during constant energy MD simulations (unless the time step size is substantially reduced). Removing the discontinuous second derivatives using a cubic spline cutoff approach, which results in almost zero changes in the characteristics of the potentials, resolves this issue. ${ }^{96}$

The $\Phi_{2 \sigma}^{i}$ and $\Phi_{2 \sigma}^{j}$ terms used in Eq. (6) have the same formulation except that they are evaluated at the center of atom $i$ and atom $j$, respectively. Hence, we discuss only $\Phi_{2 \sigma}^{i}$. Performing calculations using Eq. (6) requires the knowledge of only the product $\beta_{\sigma, i j}^{2}\left(r_{i j}\right) \cdot \Phi_{2 \sigma}^{i}$. Correspondingly, $\beta_{\sigma, i j}^{2}\left(r_{i j}\right) \cdot \Phi_{2 \sigma}^{i}$ is expressed as

$$
\beta_{\sigma, i j}^{2}\left(r_{i j}\right) \cdot \Phi_{2 \sigma}^{i}=\sum_{\substack{k=i_{1} \\ k \neq j}}^{i_{N}} g_{\sigma, j i k}^{2}\left(\theta_{j i k}\right) \cdot \beta_{\sigma, i k}^{2}\left(r_{i k}\right),
$$

where $\theta_{j i k}$ is the bond angle at atom $i$ spanning atoms $j$ and $k$ and the function $g_{\sigma, j i k}\left(\theta_{j i k}\right)$ introduces angular-dependent contributions to the bonding resulting from the overlap of the

TABLE II. Point-dependent BOP parameters for CdTe.

\begin{tabular}{lccc}
\hline \hline Symbol & Term & Cd & Te \\
\hline$p_{\pi}$ & See Eq. (A11) & 0.420000 & 0.460686 \\
\hline \hline
\end{tabular}


TABLE III. Pair-dependent BOP parameters for CdTe.

\begin{tabular}{llcc}
\hline \hline Symbol & \multicolumn{1}{c}{ Term } & Cd-Cd & Te-Te \\
\hline$r_{0}$ & GSP reference radius $(\AA)$ & 3.1276 & 3.1626 \\
$r_{c}$ & GSP characteristic radius $(\AA)$ & 3.1276 & 3.1626 \\
$r_{1}$ & Cutoff start radius $(\AA)$ & 3.7303 & 3.8046 \\
$r_{\text {cut }}$ & Cutoff radius $(\AA)$ & 4.3330 & 4.4465 \\
$n_{c}$ & GSP decay exponent & 2.800000 & 2.799998 \\
$m$ & GSP attractive exponent & 3.263155 & 2.458846 \\
$n$ & GSP repulsive exponent & 1.553883 & 1.223306 \\
$\phi_{0}$ & Repulsive energy prefactor $(\mathrm{eV})$ & 0.186369 & 0.876912 \\
$\beta_{\sigma, 0}$ & $\sigma$ Bond integral prefactor $(\mathrm{eV})$ & 0.238318 & 0.782635 \\
$\beta_{\pi, 0}$ & $\pi$ Bond integral prefactor $(\mathrm{eV})$ & 0.097599 & 0.531205 \\
$c_{\sigma}$ & Empirical $\Theta_{\sigma}$ parameter & 0.561130 & 1.014809 \\
$f_{\sigma}$ & Band-filling parameter & 0.431863 & 0.331227 \\
$k_{\sigma}$ & Skewing prefactor & 15.00000 & -2.86019 \\
$c_{\pi}$ & Empirical $\Theta_{\pi}$ parameter & 1 & 1 \\
$a_{\pi}$ & Prefactor for $\Theta_{\pi}$ & 1 & 1.8000 \\
\hline
\end{tabular}

hybridized atomic orbital. The three-body angular function is written as

$$
\begin{aligned}
g_{\sigma, j i k}\left(\theta_{j i k}\right) & \\
= & \frac{\left(b_{\sigma, j i k}-g_{0, j i k}\right) \cdot u_{\sigma, j i k}^{2}-\left(g_{0, j i k}+b_{\sigma, j i k}\right) \cdot u_{\sigma, j i k}}{2 \cdot\left(1-u_{\sigma, j i k}^{2}\right)} \\
& +\frac{g_{0, j i k}+b_{\sigma, j i k}}{2} \cdot \cos \theta_{j i k} \\
& +\frac{g_{0, j i k}-b_{\sigma, j i k}+\left(g_{0, j i k}+b_{\sigma, j i k}\right) \cdot u_{\sigma, j i k}}{2 \cdot\left(1-u_{\sigma, j i k}^{2}\right)} \cdot \cos ^{2} \theta_{j i k}
\end{aligned}
$$

where $g_{0, j i k}, b_{\sigma, j i k}$, and $u_{\sigma, j i k}$ are three-body-dependent parameters. Equation (A5) is a parabolic function exactly as previously derived ${ }^{27}$ except that the polynomial coefficients are reconstructed to satisfy $g_{\sigma, j i k}\left(\theta_{j i k}=0^{\circ}\right)=g_{0, j i k}$, $g_{\sigma}\left(\theta_{j i k}=180^{\circ}\right)=-b_{\sigma, j i k}$, and $g_{\sigma, j i k}\left(\cos \theta_{j i k}=u_{\sigma, j i k}\right)=0$. Equation (A5) can represent different orbitals. For instance, $s$ orbitals have no angular dependence so that $g_{0, j i k}=0, b_{\sigma, j i k}=$ 0 , and $u_{\sigma, j i k}= \pm 1$ (to avoid the divide-by-zero scenario, we can choose $g_{0, j i k}=0.0001, b_{\sigma, j i k}=0.0001$, and $u_{\sigma, j i k}=$ 1.00005 or -0.99995$)$; $p$ orbitals have a cosine form so that $g_{0, j i k}=1, b_{\sigma, j i k}=1$, and $u_{\sigma, j i k}=0$; and $d_{z^{2}}$ orbital have a $\cos ^{2} \theta($ or $1+\cos 2 \theta)$ dependence so that $g_{0, j i k}=1, b_{\sigma, j i k}=$ -1 , and $u_{\sigma, j i k}=0$. The three-body interactions overlap in the hybridized atomic orbitals of CdTe to form a combination of these basic angular dependences ${ }^{54}$ so that $g_{0, j i k}, b_{\sigma, j i k}$, and $u_{\sigma, j i k}$ are fitting parameters. These parameters are treated as three-body dependent to allow incorporation of asymmetry in heteroatom bonding environments. ${ }^{38,44}$

To perform calculations using Eq. (7), the product $\beta_{\sigma, i j}^{2}\left(r_{i j}\right) \cdot R_{3 \sigma, i j}$ is required. This is expressed as ${ }^{27,42}$

$$
\begin{aligned}
& \beta_{\sigma, i j}^{2}\left(r_{i j}\right) \cdot R_{3 \sigma, i j} \\
& =\sum_{\substack{k=i_{1} \\
k, j=n}}^{i_{N}} g_{\sigma}\left(\theta_{j i k}\right) \cdot g_{\sigma}\left(\theta_{i j k}\right) \cdot g_{\sigma}\left(\theta_{i k j}\right) \cdot \beta_{\sigma, i k}\left(r_{i k}\right) \cdot \beta_{\sigma, j k}\left(r_{j k}\right),
\end{aligned}
$$

where $k, j=n$ in the summation indicates that $k$ and $j$ are neighbors. The symmetric band-filling function $\Theta_{s, i j}$ was expressed as a spline function. ${ }^{27,42}$ However, in order to have continuous derivatives we have replaced the spline by the following function:

$$
\Theta_{s, i j}\left(\Theta_{\sigma, i j}^{(1 / 2)}, f_{\sigma, i j}\right)=\frac{\Theta_{0}+\Theta_{1}+S \cdot \Theta_{\sigma, i j}^{(1 / 2)}-\sqrt{\left(\Theta_{0}+\Theta_{1}+S \cdot \Theta_{\sigma, i j}^{(1 / 2)}\right)^{2}-4\left(-\varepsilon \sqrt{1+S^{2}}+\Theta_{0} \cdot \Theta_{1}+S \cdot \Theta_{1} \cdot \Theta_{\sigma, i j}^{(1 / 2)}\right)}}{2}
$$

TABLE IV. Three-body-dependent BOP parameters for CdTe.

\begin{tabular}{llllllcr}
\hline \hline \multirow{2}{*}{ Symbol } & & \multicolumn{3}{c}{$j i k$} \\
\cline { 3 - 7 } & Term & CdCdCd & TeTeTe & TeCdTe & CdCdTe & CdTeCd \\
\hline$g_{0}$ & See Eq. (11) & 1 & 1 & 1 & 1 & 1 & CdTeTe \\
$b_{\sigma}$ & See Eq. (11) & 0.762039 & 0.669623 & 0.200000 & 1.000000 & 0.2000000 & 0.999854 \\
$u_{\sigma}$ & See Eq. (11) & -0.40000 & -0.14152 & -0.38336 & 0.099711 & -0.400000 & -0.00393 \\
\hline \hline
\end{tabular}


TABLE V. Bounds on BOP parameters.

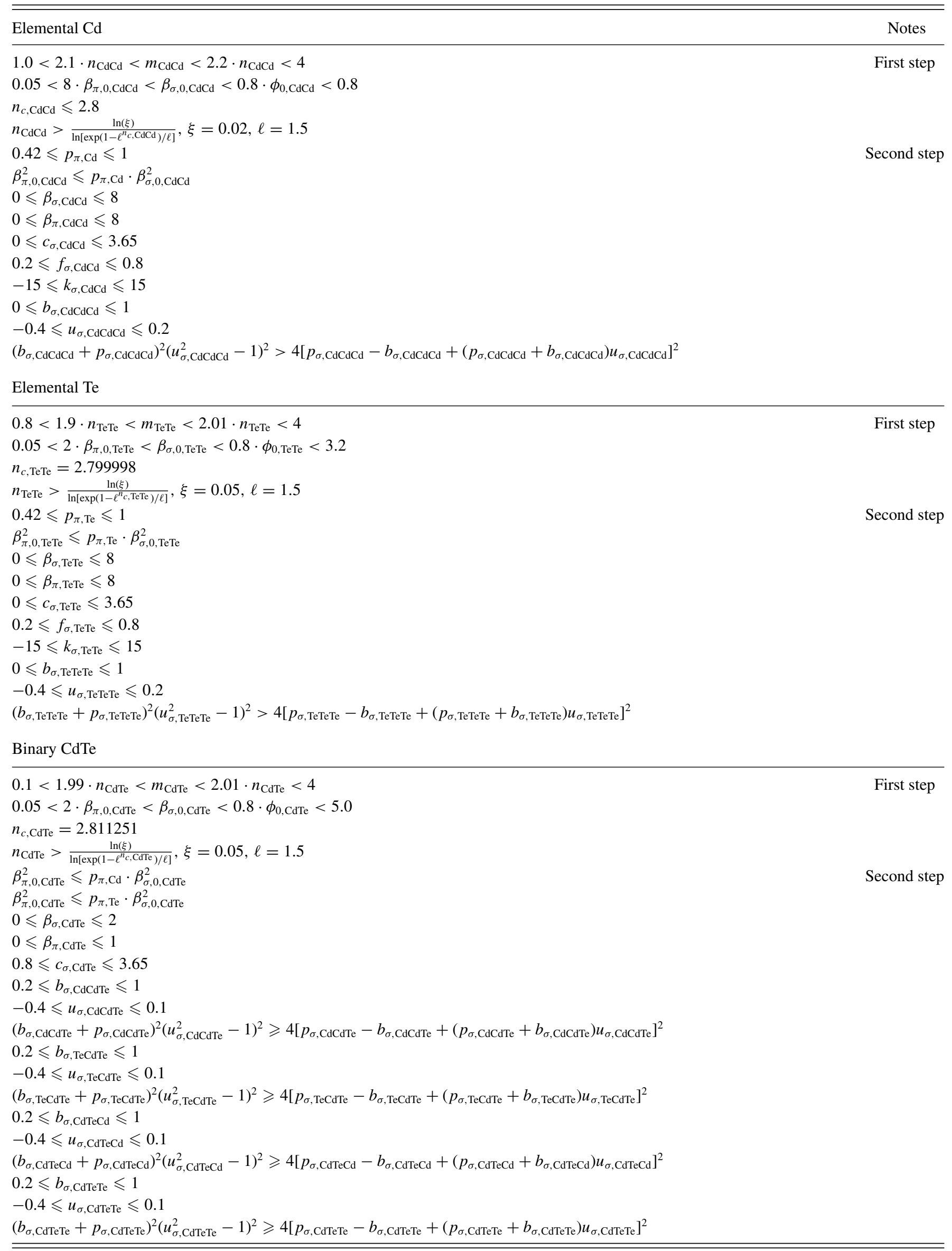


TABLE VI. Binding energies (eV/atom) for selected $\mathrm{Cd}$, Te, and CdTe clusters.

\begin{tabular}{lccccc}
\hline \hline Cluster type & DFT & BOP & SW & TR & Exp. \\
\hline Cd-di & -0.089 & -0.356 & -0.29 & -0.36 & $-0.04^{\mathrm{a}}$ \\
Cd-tetra & -0.295 & -0.633 & -0.38 & -0.33 & - \\
Te-di & -1.790 & -1.415 & -0.56 & -0.6 & $-2.66^{\mathrm{b}}$ \\
Te-rhom & -2.136 & -1.462 & -1.045 & -1.0 & - \\
CdTe-rhom & -1.306 & -1.057 & -0.952 & -0.993 & - \\
\hline \hline
\end{tabular}

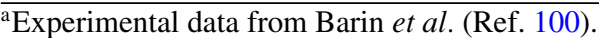

${ }^{\mathrm{b}}$ Experimental data from Viswanathan et al. (Ref. 101).

where

$$
\begin{aligned}
\varepsilon= & 10^{-10} \\
\Theta_{0}= & 15.737980 \cdot\left(\frac{1}{2}-\left|f_{\sigma, i j}-\frac{1}{2}\right|\right)^{1.137622} \\
& \cdot\left|f_{\sigma, i j}-\frac{1}{2}\right|^{2.087779} \\
S= & 1.033201 \cdot\{1-\exp [-22.180680 \\
& \left.\left.\cdot\left(\frac{1}{2}-\left|f_{\sigma, i j}-\frac{1}{2}\right|\right)^{2.689731}\right]\right\} \\
\Theta_{1}= & 2 \cdot\left(\frac{1}{2}-\left|f_{\sigma, i j}-\frac{1}{2}\right|\right) .
\end{aligned}
$$

Again, we point out that $\Theta_{\sigma, i j}^{(1 / 2)}$ is a function of the local environment around atoms $i$ and $j$ as defined by Eq. (6). The previous splined function 27,42 and Eq. (A7) are compared in Fig. 11 as a function of $\Theta_{\sigma, i j}^{(1 / 2)}$ at different $f_{\sigma, i j}$ values. It can be seen that Eq. (A7) is essentially identical to the splined function but does not suffer from the problem of discontinuous high-order derivatives at the spline junctions.

The $\beta_{\pi, i j}^{2}\left(r_{i j}\right) \cdot \Phi_{2 \pi, i j}^{i}$ and $\beta_{\pi, i j}^{4}\left(r_{i j}\right) \cdot \Phi_{4 \pi, i j}$ terms used in Eq. (8) can be written as

$$
\begin{aligned}
\beta_{\pi, i j}^{2}\left(r_{i j}\right) \cdot \Phi_{2 \pi, i j}^{i}= & \sum_{\substack{k=i_{1} \\
k \neq j}}^{i_{N}}\left[p_{\pi, i} \cdot \beta_{\sigma, i k}^{2}\left(r_{i k}\right) \cdot \sin ^{2} \theta_{j i k}\right. \\
& \left.+\left(1+\cos ^{2} \theta_{j i k}\right) \cdot \beta_{\pi, i k}^{2}\left(r_{i k}\right)\right]
\end{aligned}
$$

$$
\begin{aligned}
& \beta_{\pi, i j}^{4}\left(r_{i j}\right) \cdot \Phi_{4 \pi, i j} \\
& =\frac{1}{4} \sum_{\substack{k=i_{1} \\
k \neq j}}^{i_{N}} \sin ^{4} \theta_{j i k} \cdot \hat{\beta}_{i k}^{4}\left(r_{i k}\right)+\frac{1}{4} \sum_{\substack{k=j_{1} \\
k \neq i}}^{j_{N}} \sin ^{4} \theta_{i j k} \cdot \hat{\beta}_{j k}^{4}\left(r_{j k}\right)
\end{aligned}
$$

$$
\begin{aligned}
& +\frac{1}{2} \sum_{\substack{k=i_{1} \\
k \neq j}}^{i_{N}} \sum_{\substack{k^{\prime}=k+1 \\
k^{\prime} \neq j}}^{i_{N}} \sin ^{2} \theta_{j i k} \cdot \sin ^{2} \theta_{j i k^{\prime}} \cdot \hat{\beta}_{i k}^{2}\left(r_{i k}\right) \\
& \cdot \hat{\beta}_{i k^{\prime}}^{2}\left(r_{i k^{\prime}}\right) \cdot \cos \left(\Delta \psi_{k k^{\prime}}\right)+\frac{1}{2} \sum_{\substack{k=j_{1} \\
k \neq i}}^{j_{N}} \sum_{\substack{k^{\prime}=k+1 \\
k^{\prime} \neq i}}^{j_{N}} \sin ^{2} \theta_{i j k} \cdot \sin ^{2} \theta_{i j k^{\prime}} \\
& \cdot \hat{\beta}_{j k}^{2}\left(r_{j k}\right) \cdot \hat{\beta}_{j k^{\prime}}^{2}\left(r_{j k^{\prime}}\right) \cdot \cos \left(\Delta \psi_{k k^{\prime}}\right) \\
& +\frac{1}{2} \sum_{\substack{k^{\prime}=i_{1} \\
k^{\prime} \neq j}}^{i_{N}} \sum_{\substack{k=j_{1} \\
k \neq i}}^{j_{N}} \sin ^{2} \theta_{j i k^{\prime}} \cdot \sin ^{2} \theta_{i j k} \cdot \hat{\beta}_{i k^{\prime}}^{2}\left(r_{i k^{\prime}}\right) \\
& \cdot \hat{\beta}_{j k}^{2}\left(r_{j k}\right) \cdot \cos \left(\Delta \psi_{k k^{\prime}}\right)
\end{aligned}
$$

with

$$
\hat{\beta}_{i k}^{2}\left(r_{i k}\right)=p_{\pi, i} \cdot \beta_{\sigma, i k}^{2}\left(r_{i k}\right)-\beta_{\pi, i k}^{2}\left(r_{i k}\right),
$$

where $p_{\pi, i}$ is a species-dependent parameter of the central atom $i$. The $\beta_{\pi, i j}^{4}\left(r_{i j}\right) \cdot \Phi_{4 \pi, i j}$ term contains four-body dihedral angles $\Delta \psi_{k k^{\prime}}$ important in $\pi$ bonding. The dihedral angle is

\begin{tabular}{|c|c|c|c|c|c|c|c|c|}
\hline \multirow[b]{2}{*}{ Structure } & \multicolumn{2}{|c|}{ DFT } & \multicolumn{2}{|c|}{ BOP } & \multicolumn{2}{|c|}{ SW } & \multicolumn{2}{|c|}{ TR } \\
\hline & $r(\AA)$ & $\theta\left(^{\circ}\right)$ & $r(\AA)$ & $\theta\left(^{\circ}\right)$ & $r(\AA)$ & $\theta\left({ }^{\circ}\right)$ & $r(\AA)$ & $\theta\left(^{\circ}\right)$ \\
\hline di & 3.46 & - & $\begin{array}{l}2.75 \\
4.07^{\mathrm{a}}\end{array}$ & - & 2.98 & - & 2.92 & - \\
\hline tri & 3.39 & 60.0 & 2.86 & 60 & 3.28 & 60.0 & 3.18 & 60.0 \\
\hline $\mathrm{sq}$ & 3.44 & 90.0 & 2.85 & 90 & 3.04 & 90.0 & 3.01 & 90.0 \\
\hline tetra & 3.34 & 60.0 & 2.93 & 60.0 & 3.44 & 60.0 & 3.33 & 60.0 \\
\hline $\mathrm{ch}$ & $3.43,3.40$ & 180.0 & $2.82,2.93$ & 180.0 & $3.07,3.25$ & 180.0 & $2.94,2.96$ & 180.0 \\
\hline
\end{tabular}
defined in Fig. 12, and can be calculated as

$$
\cos \left(\Delta \psi_{k k^{\prime}}\right)=\left\{\begin{array}{l}
\frac{2\left(\cos \theta_{k i k^{\prime}}-\cos \theta_{j i k^{\prime}} \cdot \cos \theta_{j i k}\right)^{2}}{\sin ^{2} \theta_{j i k} \cdot \sin ^{2} \theta_{j i k^{\prime}}}-1 \\
\frac{2\left(\frac{i \vec{k}^{\prime} \cdot \overrightarrow{j k}}{\left|i \vec{k}^{\prime}\right| \cdot|\overrightarrow{j k}|}+\cos \theta_{i j k} \cdot \cos \theta_{j i k^{\prime}}\right)^{2}}{\sin ^{2} \theta_{i j k} \cdot \sin ^{2} \theta_{j i k^{\prime}}}-1
\end{array} .\right.
$$

\section{APPENDIX B: BOP PARAMETERS}

BOP parameters are listed in Tables I through IV for global, point-dependent, pair-dependent, and three-body-dependent parameters, respectively.

\section{APPENDIX C: DERIVATION OF THE TWO-STEP FITTING METHOD FOR BOP}

For the first step, consider some simple phases where only the nearest-neighbor atomic interactions contribute to the properties (e.g., dimer, trimer, tetra, dc, sc, fcc, gra, A8, zb, wz, $\mathrm{NaCl}$, etc.). It is important to note that all bonds within each phase are equivalent. Based upon Eqs. (1)-(4), the bond

TABLE VII. Geometries of selected Cd clusters.

${ }^{\mathrm{a}}$ Experimental data from Lukeš et al. (Ref. 102). 
TABLE VIII. Geometries of selected Te clusters.

\begin{tabular}{|c|c|c|c|c|c|c|c|c|}
\hline \multirow[b]{2}{*}{ Structure } & \multicolumn{2}{|c|}{ DFT } & \multicolumn{2}{|c|}{ BOP } & \multicolumn{2}{|c|}{ SW } & \multicolumn{2}{|c|}{ TR } \\
\hline & $r(\AA)$ & $\theta\left(^{\circ}\right)$ & $r(\AA)$ & $\theta\left({ }^{\circ}\right)$ & $r(\AA)$ & $\theta\left({ }^{\circ}\right)$ & $r(\AA)$ & $\theta\left({ }^{\circ}\right)$ \\
\hline di & 2.60 & - & $\begin{array}{c}2.74 \\
2.56^{\mathrm{a}}\end{array}$ & - & 2.86 & - & 2.78 & - \\
\hline tri & 2.77 & 60.0 & 3.01 & 60.0 & 3.11 & 60.0 & 3.01 & 60.0 \\
\hline sq & 2.81 & 90.0 & 2.99 & 90.0 & 2.90 & 90.0 & 2.86 & 90.0 \\
\hline ch & $2.60,3.15$ & 180.0 & $2.74,4.07$ & 180.0 & $3.07,3.24$ & 180.0 & $2.94,2.97$ & 180.0 \\
\hline
\end{tabular}

${ }^{a}$ Experimental data from Huber et al. (Ref. 103).

energy $E_{b, i j}$ between atoms $i$ and $j$ as a function of interatomic spacing $r_{i j}$ can be written as

$$
\begin{aligned}
E_{b, i j}= & \phi_{i j}\left(r_{i j}\right)-\beta_{\sigma, i j}\left(r_{i j}\right) \\
& \cdot \frac{2 \cdot \beta_{\sigma, 0, i j} \cdot \Theta_{\sigma, i j}+2 \cdot \beta_{\pi, 0, i j} \cdot \Theta_{\pi, i j}}{\beta_{\sigma, 0, i j}} .
\end{aligned}
$$

Note that we do not drop the subscript $i j$ even though the bonds are equivalent in a given structure because $i j$ can indicate atom types. During application of a hydrostatic strain, the bond angles do not change. For structures that have only the nearestneighbor interactions, the bond integrals only correspond to nearest neighbors and therefore are equal. It can then be seen from Eqs. (6)-(8) and (A4)-(A11) that the bond orders $\Theta_{\sigma, i j}$ and $\Theta_{\pi, i j}$ remain constant. Using the equilibrium condition $E_{b, i j}^{\prime}=0$, we have

$$
2 \cdot \beta_{\sigma, 0, i j} \cdot \Theta_{\sigma, i j}+2 \cdot \beta_{\pi, 0, i j} \cdot \Theta_{\pi, i j}=\beta_{\sigma, 0, i j} \cdot \frac{\phi_{i j}^{\prime}\left(r_{i j}\right)}{\beta_{\sigma, i j}^{\prime}\left(r_{i j}\right)} .
$$

Substituting Eq. (C2) in Eq. (C1), we have an expression of equilibrium bond energy as a function of equilibrium bond length for different phases:

$$
E_{b, i j}\left(r_{i j}\right)=\phi_{i j}\left(r_{i j}\right)-\beta_{\sigma, i j}\left(r_{i j}\right) \cdot \frac{\phi_{i j}^{\prime}\left(r_{i j}\right)}{\beta_{\sigma, i j}^{\prime}\left(r_{i j}\right)} .
$$

Similarly, we can write the second derivative of the bond energy with respect to the bond length at the equilibrium bond length using Eqs. (C1) and (C2) as

$$
E_{b, i j}^{\prime \prime}=\phi_{i j}^{\prime \prime}\left(r_{i j}\right)-\beta_{\sigma, i j}^{\prime \prime}\left(r_{i j}\right) \cdot \frac{\phi_{i j}^{\prime}\left(r_{i j}\right)}{\beta_{\sigma, i j}^{\prime}\left(r_{i j}\right)} .
$$

For the nearest-neighbor structures (with neighbor species $i j$ ), the target properties such as atomic volume $V_{i j}$, cohesive energy $E_{c, i j}$, and bulk modulus (or equivalent properties for clusters) $B_{i j}$, can be expressed using bond length $r_{i j}$, bond energy $E_{b, i j}$, and second derivatives of bond energy with respect to bond length $E_{b, i j}^{\prime \prime}$ respectively:

$$
\begin{aligned}
V_{i j} & =F \cdot r_{i j}^{3}, \quad E_{c, i j}=\frac{Z \cdot E_{b, i j}}{2}, \\
B_{i j}\left(r_{i j}\right) & =\frac{Z \cdot E_{b, i j}^{\prime \prime}}{18 F \cdot r_{i j}},
\end{aligned}
$$

where $Z$ is the atomic coordination and $F$ is a structural volume factor. Note that Eq. (C5) is applicable for both lattices and clusters without losing generality. The coordinations $Z$ for dimer, trimer, tetrahedron, dc, sc, bcc, and fcc structures are, for example, $1,2,3,4,6,8$, and 12 respectively, and $F$ takes a value of 1 for sc, $8 \sqrt{3} / 9$ for $\mathrm{dc}, \sqrt{2} / 2$ for fcc, and $4 \sqrt{3} / 9$ for bcc structures, etc. The volume for clusters is not well defined, so we simply define $F=1$ for dimer, trimer, and tetramer clusters, which means that we take $V=r_{i j}^{3}$ for clusters.

In general, when the target bond lengths, bond energies (cohesive energies), and second derivatives of the bond energy (bulk moduli) of the nearest-neighbor structures satisfy Eqs. (C2)-(C4), BOP will exactly capture these target properties. A necessary condition for this to happen is that the nearest-neighbor structures satisfy Eqs. (C3) and (C4), which involve only pairwise GSP functions. Hence, the target values of bond energies and bulk moduli of a wide range of nearest-neighbor structures with different equilibrium bond

\begin{tabular}{|c|c|c|c|c|c|c|c|c|}
\hline \multirow[b]{2}{*}{ Structure } & \multicolumn{2}{|c|}{ DFT } & \multicolumn{2}{|c|}{ BOP } & \multicolumn{2}{|c|}{ SW } & \multicolumn{2}{|c|}{$\mathrm{TR}$} \\
\hline & $r(\AA)$ & $\theta\left(^{\circ}\right)$ & $r(\AA)$ & $\theta\left(^{\circ}\right)$ & $r(\AA)$ & $\theta\left(^{\circ}\right)$ & $r(\AA)$ & $\theta\left({ }^{\circ}\right)$ \\
\hline $\mathrm{di}$ & 2.61 & - & 2.92 & - & 2.82 & - & 2.77 & - \\
\hline $\mathrm{Cd}_{2}$ Te-tri & $2.81,3.47$ & $76.4,51.8$ & $2.94,4.94$ & $114.0,33.0$ & $2.82,4.60$ & $109.5,35.3$ & $2.77,4.96$ & $126.7,26.6$ \\
\hline $\mathrm{Te}_{2}$ Cd-tri & $4.07,2.59$ & $37.1,71.0$ & $4.63,2.74$ & $34.4,72.8$ & $3.12,3.10$ & $59.6,60.4$ & $2.91,3.01$ & $62.4,58.8$ \\
\hline rhom $^{\mathrm{a}}$ & $2.77,2.77$ & $63.6,116.4$ & $3.11,3.11$ & $56.8,123.2$ & $2.88,2.88$ & $88.8,91.2$ & $2.85,2.85$ & $79.2,100.8$ \\
\hline
\end{tabular}
lengths can be used to fit Eqs. (C3) and (C4) to determine the pairwise GSP parameters $\phi_{0}, m, n$, and $n_{c}$. This firststep parameterization is similar to that used for the Tersoff potentials, ${ }^{48}$ although in the latter case all pairwise energy functions are fully determined, whereas here the parameters

TABLE IX. Geometries of selected CdTe clusters.

${ }^{\mathrm{a}}$ For rhom, the first angle $\theta$ centers at $\mathrm{Cd}$, and the second one centers at Te. 
TABLE X. Cd bulk structure properties.

\begin{tabular}{|c|c|c|c|c|c|c|c|c|}
\hline \multirow[b]{2}{*}{ Structure } & \multicolumn{2}{|c|}{ DFT } & \multicolumn{2}{|c|}{ BOP } & \multicolumn{2}{|c|}{ SW } & \multicolumn{2}{|c|}{ TR } \\
\hline & $a, c(\AA)$ & $E_{c}(\mathrm{eV})$ & $a, c(\AA)$ & $E_{c}(\mathrm{eV})$ & $a, c(\AA)$ & $E_{c}(\mathrm{eV})$ & $a, c(\AA)$ & $E_{c}(\mathrm{eV})$ \\
\hline $\mathrm{dc}$ & 6.59 & -0.679 & 6.87 & -0.725 & 6.89 & -1.160 & 6.88 & -1.159 \\
\hline $\mathrm{sc}$ & 2.96 & -1.086 & 3.12 & -0.618 & 3.34 & -0.952 & 3.24 & -0.870 \\
\hline bcc & 3.61 & -1.351 & 3.54 & -1.019 & 4.04 & -0.916 & 3.96 & -0.801 \\
\hline fcc & 4.60 & -1.402 & 4.45 & -1.128 & 5.16 & -0.893 & 4.96 & -0.871 \\
\hline hcp & $3.12,5.58$ & -1.398 & $\begin{array}{c}3.16,5.11 \\
(2.97,5.61)^{a}\end{array}$ & $\begin{array}{l}-1.135 \\
-1.133^{b}\end{array}$ & $3.12,5.95$ & -0.893 & $3.01,5.73$ & -0.871 \\
\hline gra & $4.89,5.31$ & -0.946 & $5.01,5.76$ & -0.671 & $5.27,6.75$ & -0.934 & $5.07,7.60$ & -1.064 \\
\hline grap & 5.31 & -0.340 & 5.10 & -0.599 & 5.21 & -0.829 & 5.07 & -1.053 \\
\hline
\end{tabular}

${ }^{\text {a Experimental data (Ref. 72). }}$

${ }^{\mathrm{b}}$ Experimental data (Ref. 71).

$\beta_{\sigma, 0}$ and $\beta_{\pi, 0}$ cannot be determined because they cancel out and do not appear in Eqs. (C3) and (C4).

As Eqs. (C3) and (C4) are necessary conditions for fitting the target properties, Eq. (C2) must also be satisfied for the predicted properties to match the targets. The parameters determined in the first step enable evaluations of the right-hand side of Eq. (C2) at the target equilibrium bond lengths for different nearest-neighbor structures. This creates a new set of target values for the combined bond-order term $2 \cdot \beta_{\sigma, 0, i j} \cdot \Theta_{\sigma, i j}+2 \cdot \beta_{\pi, 0, i j} \cdot \Theta_{\pi, i j}$. These target values, along with target properties (cohesive energies, lattice constants, etc.) of non-nearest-neighbor structures, can then be fitted in a second step to determine the remaining parameters, $p_{\pi}, \beta_{\sigma, 0}$, $\beta_{\pi, 0}, c_{\sigma}, f_{\sigma}, k_{\sigma}, b_{\sigma}$, and $u_{\sigma}$.

\section{APPENDIX D: PARAMETER BOUNDS}

The parameters were bounded with physical ranges during parameterizations, and these constraints are listed in Table V in six groups representing first- and second-step parameterizations of $\mathrm{Cd}$, Te, and CdTe, respectively.

\section{APPENDIX E: DFT METHOD}

Our DFT results for the various CdTe small clusters, bulk lattices, point defects, and surfaces were based on spin- polarized, generalized gradient approximation (GGA) methods using projector-augmented-wave (PAW) pseudopotentials with a dispersion-corrected Perdew-Burke-Ernzerhof (PBED2) functional. ${ }^{97}$ During our study, we found that the inclusion of dispersion effects in the CdTe systems was essential for obtaining accurate cohesive energies and bulk moduli (especially for the more weakly bound Cd clusters). Within the DFT-D2 approach, ${ }^{98,99}$ an atomic pairwise dispersion correction is added to the Kohn-Sham part of the total energy $\left(E_{\mathrm{KS}-\mathrm{DFT}}\right)$ as

$$
E_{\mathrm{DFT}-\mathrm{D}}=E_{\mathrm{KS}-\mathrm{DFT}}+E_{\mathrm{disp}},
$$

where $E_{\text {disp }}$ is given by

$$
E_{\mathrm{disp}}=-s_{6} \sum_{i=1}^{N_{\mathrm{at}}-1} \sum_{j=i+1}^{N_{\mathrm{at}}} \sum_{\mathbf{g}} f_{\mathrm{damp}}\left(R_{i j, \mathbf{g}}\right) \frac{C_{6}^{i j}}{R_{i j, \mathbf{g}}^{6}} .
$$

Here, the summation is over all atom pairs $i$ and $j$, and over all $\mathbf{g}$ lattice vectors with the exclusion of the $i=j$ contribution when $\mathbf{g}=0$ (this restriction prevents atomic self-interaction in the reference cell). The parameter $C_{6}^{i j}$ is the dispersion coefficient for atom pairs $i$ and $j$, calculated as the geometric mean of the atomic dispersion coefficients: $C_{6}^{i j}=\sqrt{C_{6}^{i} C_{6}^{j}}$.

The $s_{6}$ parameter is a global scaling factor, which is specific to the adopted DFT method ( $s_{6}=0.75$ for PBE), and $R_{i j}, \mathrm{~g}$ is

\begin{tabular}{|c|c|c|c|c|c|c|c|c|}
\hline \multirow[b]{2}{*}{ Structure } & \multicolumn{2}{|c|}{ DFT } & \multicolumn{2}{|c|}{ BOP } & \multicolumn{2}{|c|}{ SW } & \multicolumn{2}{|c|}{$\mathrm{TR}$} \\
\hline & $a, c(\AA)$ & $E_{c}(\mathrm{eV})$ & $a, c(\AA)$ & $E_{c}(\mathrm{eV})$ & $a, c(\AA)$ & $E_{c}(\mathrm{eV})$ & $a, c(\AA)$ & $E_{c}(\mathrm{eV})$ \\
\hline $\mathrm{dc}$ & 7.12 & -2.272 & 7.23 & -1.943 & 6.89 & -2.230 & 6.88 & -2.120 \\
\hline $\mathrm{sc}$ & 3.17 & -2.765 & 3.23 & -2.163 & 3.34 & -2.097 & 3.24 & -2.091 \\
\hline bcc & 3.87 & -2.551 & 3.93 & -1.941 & 4.04 & -2.108 & 3.96 & -2.669 \\
\hline fcc & 4.84 & -2.399 & 4.95 & -1.846 & 5.16 & -2.054 & 4.96 & -2.624 \\
\hline A8 & $4.34,6.05$ & -2.798 & $\begin{array}{c}4.53,5.51 \\
(4.45,5.91)^{\mathrm{a}}\end{array}$ & $\begin{array}{c}-2.167 \\
-2.168^{b}\end{array}$ & $3.50,8.69$ & -2.064 & $3.61,7.91$ & -2.599 \\
\hline gra & $5.21,6.06$ & -2.468 & $5.38,6.63$ & -1.873 & $5.04,6.33$ & -1.889 & $5.13,5.21$ & -1.958 \\
\hline grap & 5.31 & -2.122 & 5.343 & -1.697 & 4.99 & -1.617 & 4.83 & -1.770 \\
\hline
\end{tabular}

TABLE XI. Te bulk structure properties.

${ }^{\mathrm{a}}$ Experimental data (Ref. 72).

${ }^{\mathrm{b}}$ Experimental data (Ref. 71). 
TABLE XII. CdTe bulk structure properties (一: not calculated).

\begin{tabular}{|c|c|c|c|c|c|c|c|c|}
\hline \multirow[b]{2}{*}{ Structure } & \multicolumn{2}{|c|}{ DFT } & \multicolumn{2}{|c|}{ BOP } & \multicolumn{2}{|c|}{ SW } & \multicolumn{2}{|c|}{$\mathrm{TR}$} \\
\hline & $a, c(\AA)$ & $E_{c}(\mathrm{eV})$ & $a-c(\AA)$ & $E_{c}(\mathrm{eV})$ & $a-c(\AA)$ & $E_{c}(\mathrm{eV})$ & $a-c(\AA)$ & $E_{c}(\mathrm{eV})$ \\
\hline B1 & 6.04 & -2.287 & 6.19 & -2.140 & 6.35 & -1.796 & 5.85 & -2.177 \\
\hline B2 & 3.81 & -2.006 & 3.83 & -1.656 & 3.94 & -1.719 & 3.63 & -2.339 \\
\hline wz & $4.52,7.32$ & -2.279 & $4.84,7.88$ & -2.173 & $3.98,7.51$ & -2.060 & $3.97,7.49$ & -2.060 \\
\hline $\mathrm{zb}$ & 6.52 & -2.331 & $\begin{array}{c}6.83 \\
6.48^{\mathrm{a}}\end{array}$ & $\begin{array}{c}-2.173 \\
-2.178^{b}\end{array}$ & 6.51 & -2.060 & 6.49 & -2.060 \\
\hline fcs & 4.10 & -1.807 & 4.28 & -1.684 & 4.39 & -1.343 & 4.06 & -1.696 \\
\hline grap & 4.75 & -1.916 & 5.11 & -1.643 & 4.92 & -1.485 & 4.81 & -1.690 \\
\hline $\mathrm{NiAs}$ & - & - & $4.30,7.54$ & -2.009 & $4.37,7.91,7.57$ & -1.766 & $3.72,8.33,6.45$ & -2.310 \\
\hline $\mathrm{L}_{1}$ & - & - & $4.37,10.74$ & -2.140 & $4.49,11.0,7.78$ & -1.796 & $3.72,14.50,6.45$ & -2.310 \\
\hline $\mathrm{L}_{0}$ & - & - & $5.39,7.64$ & -1.656 & $5.57,7.88$ & -1.719 & $5.13,7.24$ & -2.339 \\
\hline sc16 & - & - & 8.38 & -1.974 & 8.03 & -1.871 & not stable & not stable \\
\hline $\mathrm{Cd}_{2} \mathrm{Te}-\mathrm{Ag}_{2} \mathrm{O}$ & - & - & 6.86 & -1.384 & 6.74 & -1.175 & 6.48 & -1.387 \\
\hline $\mathrm{Te}_{2} \mathrm{Cd}-\mathrm{Ag}_{2} \mathrm{O}$ & - & - & 6.86 & -1.387 & 6.74 & -1.175 & 6.48 & -1.387 \\
\hline $\mathrm{Cd}_{2} \mathrm{Te}-\mathrm{ZrO}_{2}$ & - & - & 7.39 & -1.693 & 7.46 & -1.775 & 7.00 & -2.395 \\
\hline $\mathrm{Te}_{2} \mathrm{Cd}-\mathrm{ZrO}_{2}$ & - & - & 7.29 & -1.632 & 7.58 & -1.565 & 7.00 & -1.828 \\
\hline
\end{tabular}

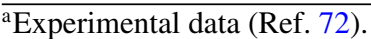

${ }^{\mathrm{b}}$ Experimental data (Ref. 71).

the interatomic distance between atom $i$ in the reference cell and $j$ in the neighboring cell at distance $|\mathbf{g}|$. A cutoff distance of $30.0 \AA$ was used to truncate the lattice summation. In order to avoid near-singularities for small interatomic distances, the damping function has the form

$$
f_{\text {damp }}\left(R_{i j, \mathbf{g}}\right)=\frac{1}{1+\exp \left[-d\left(R_{i j, \mathbf{g}} / R_{\mathrm{vdW}}-1\right)\right]},
$$

where $R_{\mathrm{vdW}}$ is the sum of atomic van der Waals radii $\left(R_{\mathrm{vdW}}=R_{\mathrm{vdW}}^{i}+R_{\mathrm{vdW}}^{j}\right)$ and $d$ controls the steepness of the damping function.

For all the small-cluster and bulk-lattice calculations, we used a very high cutoff energy of $500 \mathrm{eV}$ for the plane-wave basis set, and the Brillouin zone was sampled using a dense $10 \times 10 \times 10$ gamma-centered Monkhorst-Pack grid. In addition to spin-polarization and dispersion effects, we also included a relativistic spin-orbit coupling treatment for all the valence electrons in both the small-cluster and bulk-lattice calculations. Unconstrained geometry optimizations of both the ions and the unit cell were carried out. To prevent spurious interactions between adjacent clusters for the small-cluster

TABLE XIII. Elastic constants of zinc-blende CdTe (GPa).

\begin{tabular}{lccccc}
\hline \hline$c_{i j}$ & Expt. (300 K) & DFT & BOP & SW & TR \\
\hline$c_{11}$ & $53.3^{\mathrm{b}}$ & $53.2^{\mathrm{c}}$ & 50.7 & 44.3 & 50.7 \\
$c_{12}$ & $36.5^{\mathrm{b}}$ & $36.0^{\mathrm{c}}$ & 37.5 & 19.6 & 37.5 \\
$c_{44}$ & $20.4^{\mathrm{b}}$ & - & 16.5 & 18.0 & 15.2 \\
$c_{44^{\mathrm{a}}}$ & - & $31.8^{\mathrm{c}}$ & 30.6 & 30.7 & 46.8 \\
\hline \hline
\end{tabular}

\footnotetext{
${ }^{\text {a Unrelaxed. }}$

${ }^{\mathrm{b}}$ Data from Rowe et al. (Ref. 74).

${ }^{\mathrm{c}}$ Data from Agrawal et al. (Ref. 75).
}

calculations, the vacuum along all three axes was set to $25 \AA$ during the geometry optimization.

Because the point-defect and surface calculations require the use of larger supercells and significantly more atoms (>200 atoms), a smaller $300-\mathrm{eV}$ cutoff energy was used for both calculations. For this same reason, we did not include spin-orbit effects in these large supercell systems, although we still carried out these calculations with unconstrained spin-polarized conditions. In the point-defect calculations, a large $3 \times 3 \times 3$ supercell was used, and therefore, a smaller $2 \times 2 \times 2$ gamma-centered Monkhorst-Pack grid was used. For the surface calculations, a slab geometry was chosen, which consisted of seven layers of CdTe and 35 $\AA$ of vacuum between adjacent slabs. In these calculations, a $4 \times 4 \times 1$ gamma-centered Monkhorst-Pack grid was used. Unconstrained geometry optimizations of both the ions and the unit cell were carried out. We should also note that for a few selected configurations of our surface calculations, we observed a significant reconstruction of the surface compared to a previous DFT study, ${ }^{89}$ which used a (simpler) local-density approximation (LDA) functional. While the PBE-D2 method has been extensively tested for several bulk lattices and clusters, it is possible that the PBE-D2 dispersion parameters for $\mathrm{Cd}$ and $\mathrm{Te}$ may need further tuning
TABLE XIV. CdTe-zb melting temperature (K).

\begin{tabular}{lc}
\hline \hline Exp. $^{a}$ & 1365 \\
BOP & $1550-1600$ \\
SW & $1360-1390$ \\
TR & $700-800$ \\
\hline \hline
\end{tabular}

$\overline{\bar{a}}$ Experimental data (Ref. 79). 
TABLE XV. Intrinsic defect energies $E_{D}^{\prime}$ of various defects in CdTe-zb calculated using different models.

\begin{tabular}{lllll}
\hline \hline Defect & DFT & BOP & SW & TR \\
\hline$V_{\mathrm{Cd}}$ & 2.37 & 3.17 & 2.60 & 2.42 \\
$V_{\mathrm{Te}}$ & 0.95 & 1.12 & 1.53 & 0.93 \\
$\mathrm{Cd}_{\mathrm{Te}}$ & 2.12 & 2.21 & 0.8 & 0.18 \\
$\mathrm{Te}_{\mathrm{Cd}}$ & 3.71 & 3.06 & 0.74 & 1.19 \\
$\mathrm{Cd}_{i}($ tetrahedral $)$ & 1.40 & 1.60 & 3.76 & 0.61 \\
$\mathrm{Te}_{i}($ tetrahedral $)$ & 2.47 & 4.16 & 2.6 & 0.55 \\
$\mathrm{Cd}_{i}(\langle 110\rangle$ dumbbell $)$ & 1.38 & 2.08 & 3.88 & 0.43 \\
$\mathrm{Te}_{i}(\langle 110\rangle$ dumbbell $)$ & 2.56 & 3.58 & 3.67 & 1.10 \\
$\mathrm{Cd}_{i}(\langle 100\rangle$ dumbbell $)$ & 2.20 & 2.81 & 2.34 & 0.48 \\
$\mathrm{Te}_{i}(\langle 100\rangle$ dumbbell $)$ & 2.84 & 3.44 & 2.69 & 2.02 \\
\hline \hline
\end{tabular}

to describe the complex electronic interactions (i.e., dangling bonds) at a free surface, which we save for future work.

\section{APPENDIX F: COMPLETE LIST OF PREDICTED PROPERTIES}

The binding energies for some selected $\mathrm{Cd}$, Te, and CdTe clusters obtained from various models and experiments in the literature ${ }^{100,101}$ are shown in Table VI. The geometries obtained from various models and experiments in the literature ${ }^{102,103}$ are shown in Tables VII-IX, respectively, for selected $\mathrm{Cd}$, Te, and CdTe clusters.

The lattice constants and cohesive energies obtained from various models and experiments in the literature ${ }^{71,72}$ are shown in Tables X-XII, respectively, for numerous $\mathrm{Cd}, \mathrm{Te}$, and (stoichiometric/nonstoichiometric) CdTe lattices.

The single crystal elastic constants of CdTe-zb obtained from various models and experiments in the literature ${ }^{74} / \mathrm{DFT}$ calculations $^{75}$ are shown in Table XIII.

Melting temperature of CdTe-zb obtained from different methods and literature experiments ${ }^{79}$ is compared in Table XIV.

The various defect energies of the CdTe-zb phase calculated using different models are compared in Table XV. *donward@sandia.gov

${ }^{1}$ L. L. Kazmerski, J. Electron Spectrosc. Relat. Phenomena 150, 105 (2006).

${ }^{2}$ J. J. Loferski, J. Appl. Phys. 27, 777 (1956).

${ }^{3}$ G. S. Khrypunov, E. P. Chernykh, N. A. Kovtun, and E. K. Belonogov, Semiconductors 43, 1046 (2009).

${ }^{4}$ R. H. Bube and K. W. Mitchell, J. Electron. Mater. 22, 17 (1993).

${ }^{5}$ H. C. Chou and A. Rohatgi, J. Electron. Mater. 23, 31 (1994).

${ }^{6}$ K. Zweibel, Science 328, 3 (1995).

${ }^{7}$ W. H. Bloss, F. Pfisterer, M. Schubert, and T. Walter, Prog. Photovolt. 3, 3 (1995).

${ }^{8}$ M. D. G. Potter, M. Cousins, K. Durose, and D. P. Halliday, J. Mater. Sci.: Mater. Electron. 11, 525 (2000).

${ }^{9}$ T. E. Schlesinger, J. E. Toney, H. Yoon, E. Y. Lee, B. A. Brunett, L. Franks, and R. B. James, Mater. Sci. Eng. 32, 103 (2001).

${ }^{10}$ P. J. Sellin, Nucl. Instrum. Methods Phys. Res. A. 513, 332 (2003).

${ }^{11}$ A. Peurrung, Mater. Today 11, 50 (2008).

${ }^{12}$ T. Takahashi and S. Watanabe, IEEE Trans. Nucl. Sci. 48, 950 (2001).

${ }^{13}$ A. Shah, P. Torres, R. Tscharner, N. Wyrsch, and H. Keppner, Science 285, 692 (1999).

${ }^{14}$ J. D. Beach and B. E. McCandless, MRS Bull. 32, 225 (2007).

${ }^{15}$ K. D. Dobson, I. Visoly-Fisher, G. Hodes, and D. Cahen, Sol. Energy Mater. Sol. Cells 62, 295 (2000).

${ }^{16}$ A. Rohatgi, R. Sudharsanan, S. A. Ringel, and M. H. MacDougal, Sol. Cells. 30, 109 (1991).

${ }^{17}$ J. Versluys, P. Clauws, P. Nollet, S. Degrave, and M. Burgelman, Thin Solid Films 451-452, 434 (2004).

${ }^{18}$ Y. Yan, M. M. Al-Jassim, and K. M. Jones, J. Appl. Phys. 94, 2976 (2003).

${ }^{19}$ A. E. Bolotnikov, G. S. Camarda, G. A. Carini, Y. Cui, L. Li, and R. B. James, Nucl. Instrum. Methods Phys. Res. A 579, 125 (2007).

${ }^{20}$ A. E. Bolotnikov, G. S. Camarda, G. A. Carini, Y. Cui, L. Li, and R. B. James, Nucl. Instrum. Methods Phys. Res. A 571, 687 (2007).

${ }^{21}$ C. Szeles, Phys. Status Solidi A 241, 783 (2004).

${ }^{22}$ C. Szeles, IEEE Trans. Nucl. Sci. 51, 1242 (2004).
${ }^{23}$ G. Zha, W. Jie, T. Tan, and L. Wang, Phys. Status Solidi A 204, 2196 (2007).

${ }^{24}$ V. Babentsov, V. Boiko, G. A. Schepelskii, R. B. James, J. Franc, J. Prochazka, and P. Hlidek, Nucl. Instrum. Methods Phys. Res. A 633, S81 (2011).

${ }^{25}$ D. Zeng, W. Jie, T. Wang, G. Zha, and J. Zhang, Nucl. Instrum. Methods Phys. Res. A 586, 439 (2008).

${ }^{26}$ D. G. Pettifor and I. I. Oleinik, Phys. Rev. B 59, 8487 (1999).

${ }^{27}$ D. A. Murdick, X. W. Zhou, H. N. G. Wadley, D. Nguyen-Manh, R. Drautz, and D. G. Pettifor, Phys. Rev. B 73, 045206 (2006).

${ }^{28}$ LAMMPS, lammps.sandia.gov/download (2011).

${ }^{29}$ S. Plimpton, J. Comput. Phys. 117, 1 (1995).

${ }^{30}$ Z. Q. Wang, D. Stroud, and A. J. Markworth, Phys. Rev. B 40, 3129 (1989).

${ }^{31}$ F. H. Stillinger and T. A. Weber, Phys. Rev. B 31, 5262 (1985).

${ }^{32}$ J. Oh and C. H. Grein, J. Cryst. Growth 193, 241 (1998).

${ }^{33}$ J. Wang and A. Rockett, Phys. Rev. B 43, 12571 (1991).

${ }^{34}$ J. Tersoff, Phys. Rev. B 39, 5566 (1989).

${ }^{35}$ D. K. Ward, X. W. Zhou, B. M. Wong, F. P. Doty, and J. A. Zimmerman, J. Chem. Phys. 134, 244703 (2011).

${ }^{36}$ D. W. Brenner, Phys. Rev. B 42, 9458 (1990).

${ }^{37}$ G. C. Abell, Phys. Rev. B 31, 6184 (1985).

${ }^{38}$ D. G. Pettifor, M. W. Finnis, D. Nguyen-Manh, D. A. Murdick, X. W. Zhou, and H. N. G. Wadley, Mater. Sci. Eng. A 365, 2 (2004).

${ }^{39}$ D. G. Pettifor and I. I. Oleinik, Phys. Rev. Lett. 84, 4124 (2000).

${ }^{40}$ D. G. Pettifor and I. I. Oleinik, Phys. Rev. B 65, 172103 (2002).

${ }^{41}$ R. Drautz, D. Nguyen-Manh, D. A. Murdick, X. W. Zhou, H. N. G. Wadley, and D. G. Pettifor, TMS Lett. 1, 31 (2004).

${ }^{42}$ R. Drautz, D. A. Murdick, D. Nguyen-Manh, X. W. Zhou, H. N. G. Wadley, and D. G. Pettifor, Phys. Rev. B 72, 144105 (2005).

${ }^{43}$ D. A. Murdick, H. N. G. Wadley, and X. W. Zhou, Phys. Rev. B 75, 125318 (2007).

${ }^{44}$ D. A. Murdick, X. W. Zhou, and H. N. G. Wadley, Phys. Rev. B 72, 205340 (2005). 
${ }^{45}$ M. Nakamura, H. Fujioka, K. Ono, M. Takeuchi, T. Mitsui, and M. Oshima, J. Cryst. Growth 209, 232 (2000).

${ }^{46}$ P. A. Ashu, J. H. Jefferson, A. G. Cullis, W. E. Hagston, and C. R. Whitehouse, J. Cryst. Growth 150, 176 (1995).

${ }^{47}$ J. Nord, K. Albe, P. Erhart, and K. Nordlund, J. Phys. Condens. Matter 15, 5649 (2003).

${ }^{48}$ K. Albe, K. Nordlund, J. Nord, and A. Kuronen, Phys. Rev. B 66, 035205 (2002).

${ }^{49}$ X. W. Zhou, D. A. Murdick, B. Gillespie, and H. N. G. Wadley, Phys. Rev. B 73, 045337 (2006).

${ }^{50}$ D. A. Murdick, X. W. Zhou, H. N. G. Wadley, R. Drautz, and D. G. Pettifor, Materials Research Society Symposium Proceedings (Materials Research Society, Warrendale, PA, 2005), p. JJ9.7.1.

${ }^{51}$ D. G. Pettifor, Phys. Rev. Lett. 63, 2480 (1989).

${ }^{52}$ A. P. Horsfield, A. M. Bratkovsky, M. Fearn, D. G. Pettifor, and M. Aoki, Phys. Rev. B 53, 12694 (1996).

${ }^{53}$ D. G. Pettifor, Springer Proc. Phys. 48, 64 (1990).

${ }^{54}$ D. G. Pettifor, Bonding and Structure of Molecules and Solids (Oxford University Press, Oxford, 1995).

${ }^{55} \mathrm{M}$. Finnis, Interatomic Forces in Condensed Matter (Oxford University Press, Oxford, 2003).

${ }^{56}$ L. Goodwin, A. J. Skinner, and D. G. Pettifor, Europhys. Lett 9, 701 (1989).

${ }^{57}$ C. Lanczos, J. Res. Natl. Bur. Stand. 45, 225 (1950).

${ }^{58}$ R. Haydock, Solid State Phys. 35, 216 (1980).

${ }^{59}$ R. Jones and M. W. Lewis, Philos. Mag. B 49, 95 (1984).

${ }^{60}$ J. Inoue and Y. Ohta, J. Phys. C 20, 1947 (1987).

${ }^{61}$ F. Cyrot-Lackmann, Adv. Phys. 16, 393 (1967).

${ }^{62}$ J. E. Lennard-Jones, Proc. R. Soc. Lond. A 106, 463 (1924).

${ }^{63}$ P. M. Morse, Phys. Rev. 34, 57 (1929).

${ }^{64} \mathrm{~S}$. Wolfram, The Mathematica Book, 5th ed. (Wolfram Research, Inc., Champaign, IL, 2004), p. $106 \mathrm{ff}$.

${ }^{65}$ M. R. Hestenes and E. Stiefel, J. Res. Natl. Bur. Stand. 49, 409 (1952).

${ }^{66}$ D. M. Olsson and L. S. Nelson, Technometrics 17, 45 (1975).

${ }^{67}$ R. Storn and K. Price, J. Global Optim. 11, 341 (1997).

${ }^{68}$ S. Kirkpatrick, C. D. Gelatt, and M. P. Vecchi, Science 220, 671 (1983).

${ }^{69}$ W. H. Press, Numerical Recipes in Fortran 77: The Art of Scientific Computing, 2nd ed. (Cambridge University Press, New York, 1996), Vol. 1.

${ }^{70}$ M. Parrinello and A. Rahman, J. Appl. Phys. 52, 7182 (1981).

${ }^{71}$ I. Barin, Thermochemical Data of Pure Substances (VCH, Weinheim, 1993).

${ }^{72}$ J. D. H. Donnay and H. M. Ondik, Crystal Data, Determinative Tables, 3rd ed. (US Department of Commerce, National Bureau of Standards, and Joint Committee on Power Diffraction Standards, Washington DC, USA, 1973), Vol. 2.

${ }^{73}$ P. Ruterana, B. Barbaray, A. Béré, P. Vermaut, A. Hairie, E. Paumier, G. Nouet, A. Salvador, A. Botchkarev, and H. Morkoç, Phys. Rev. B 59, 15917 (1999).

${ }^{74}$ J. M. Rowe, R. M. Nicklow, D. L. Price, and K. Zanio, Phys. Rev. B 10, 671 (1974).
${ }^{75}$ B. K. Agrawal and S. Agrawal, Phys. Rev. B 45, 8321 (1992).

${ }^{76}$ J. R. Morris, C. Z. Wang, K. M. Ho, and C. T. Chan, Phys. Rev. B 49, 3109 (1994).

${ }^{77}$ M. E. Tuckerman, J. Alejandre, R. López-Rendón, A. L. Jochim, and G. J. Martyna, J. Phys. A 39, 5629 (2006).

${ }^{78}$ W. Shinoda, M. Shiga, and M. Mikami, Phys. Rev. B 69, 134103 (2004).

${ }^{79}$ I. S. Grigoriev and E. Z. Meilikhov, Handbook of Physical Quantities (CRC Press, New York, 1997).

${ }^{80}$ R. D. S. Yadava, R. K. Bagai, and W. N. Borle, J. Electron Mater. 21, 1001 (1992).

${ }^{81}$ C. Szeles, IEEE Trans. Nucl. Sci. 51, 1242 (2004).

${ }^{82}$ S. B. Zhang and J. E. Northrup, Phys. Rev. Lett. 67, 2339 (1991).

${ }^{83}$ J. E. Northrup and S. B. Zhang, Phys. Rev. B 47, 6791 (1993).

${ }^{84}$ G. Zollo and R. M. Nieminen, J. Phys. Condens. Matter 15, 843 (2003).

${ }^{85}$ D. J. Chadi, Phys. Rev. B 46, 9400 (1992).

${ }^{86}$ L. Seehofer, G. Falkenberg, R. L. Johnson, V. H. Etgens, S. Tatarenko, D. Brun, and B. Daudin, Appl. Phys. Lett. 67, 1680 (1995).

${ }^{87}$ B. Daudin, D. Brun-Le Cunff, and S. Tatarenko, Surf. Sci. 352-354, 99 (1996).

${ }^{88}$ M. B. Veron, V. H. Etgens, M. Sauvage-Simkin, S. Tatarenko, B. Daudin, and D. Brun-Le Cunff, J. Cryst. Growth 159, 694 (1996).

${ }^{89}$ S. Gundel, A. Fleszar, W. Faschinger, and W. Hanke, Phys. Rev. B 59, 15261 (1999).

${ }^{90}$ S. Tatarenko, B. Daudin, D. Brun, V. H. Etgens, and M. B. Veron, Phys. Rev. B 50, 18479 (1994).

${ }^{91}$ L. Zhang and J. Y. Feng, Nucl. Instrum. Methods Phys. Res. B 234, 487 (2005).

${ }^{92}$ J. L. Xu and J. Y. Feng, Nucl. Instrum. Methods Phys. Res. B 217, 33 (2004).

${ }^{93}$ P. Beaucage and N. Mousseau, Phys. Rev. B 71, 094102 (2005).

${ }^{94}$ L. D. Landau and E. M. Lifshitz, Mechanics, 3rd ed. (Pergamon Press, New York, 1976).

${ }^{95}$ A. Nordsieck, Math. Comput. 16, 22 (1962).

${ }^{96}$ X. W. Zhou and R. E. Jones, Modell. Simul. Mater. Sci. Eng. 19, 25004 (2011)

${ }^{97}$ J. P. Perdew, K. Burke, and M. Ernzerhof, Phys. Rev. Lett. 77, 3865 (1996).

${ }^{98}$ S. Grimme, J. Comput. Chem. 27, 1787 (2006).

${ }^{99}$ B. M. Wong and S. H. Ye, Phys. Rev. B 84, 075115 (2011).

${ }^{100}$ I. Barin, O. Knacke, and O. Kubaschewski, Thermochemical Properties of Inorganic Compounds (Springer-Verlag, Berlin, 1977).

${ }^{101}$ R. Viswanathan, M. Sai Baba, D. Darwin Albert Raj, R. Balasubramanian, T. S. Lakshmi Narasimhan, and C. K. Mathews, Spectrochim. Acta, Part B 49, 243 (1994).

${ }^{102}$ V. Lukeš, M. Ilčin, V. Laurinc, and S. Biskupič, Chem. Phys. Lett. 424, 199 (2006).

${ }^{103}$ K. P. Huber and G. Herzberg, Molecular Spectra and Molecular Structure. IV. Constants for Diatomic Molecules (Van Nostrand Reinghold, New York, 1979). 